\title{
A DECOMPOSITION THEOREM OF THE MÖBIUS ENERGY I: DECOMPOSITION AND MÖBIUS INVARIANCE
}

\author{
Aya IShizeki AND TAKEYUKI NAgASAWA ${ }^{1}$
}

\begin{abstract}
The Möbius energy, defined for closed curves embedded in $\mathbf{R}^{n}$, is decomposed into three parts. It is called the Möbius energy since it is invariant under Möbius transformations. An alternative proof of the Möbius invariance is given using the decomposition. Furthermore the invariance of each part of decomposition is discussed.
\end{abstract}

\section{Introduction}

Let $\boldsymbol{f}: \mathbf{R} / \mathcal{L} \mathbf{Z} \ni s \mapsto \boldsymbol{f}(s) \in \mathbf{R}^{n}$ be a closed curve in $\mathbf{R}^{n}$ with total length $\mathcal{L}$, where $s$ is an arc-length parameter, i.e., $\left\|\boldsymbol{f}^{\prime}(s)\right\|_{\mathbf{R}^{n}} \equiv 1$. We denote the distance between $\boldsymbol{f}\left(s_{1}\right)$ and $\boldsymbol{f}\left(s_{2}\right)$ along the closed curve by $\mathscr{D}\left(\boldsymbol{f}\left(s_{1}\right), \boldsymbol{f}\left(s_{2}\right)\right)$.

O'Hara [7] defined the Möbius energy $\mathcal{E}$ as

$$
\mathcal{E}(\boldsymbol{f})=\text { p.v. } \iint\left(\frac{1}{\left\|\boldsymbol{f}\left(s_{1}\right)-\boldsymbol{f}\left(s_{2}\right)\right\|_{\mathbf{R}^{n}}^{2}}-\frac{1}{\mathscr{D}\left(\boldsymbol{f}\left(s_{1}\right), \boldsymbol{f}\left(s_{2}\right)\right)^{2}}\right) d s_{1} d s_{2},
$$

where p.v. $\iint=\lim _{\varepsilon \rightarrow+0} \iint_{\left|s_{1}-s_{2}\right|>\varepsilon}$ is Cauchy's principal value.

Remark 1.1. This is the original definition of O'Hara. In fact, the integration is not a principal value since the integrand is non-negative. However, many quantities derived from the energy, for example the variational formulae, contain terms each of which is not absolutely integrable. Therefore, when deforming the expression of the energy, we always deal with it as Cauchy's principal value at first, and investigate its absolute integrability later.

Each term of the integrand in (1) is not integrable on $(\mathbf{R} / \mathcal{L Z})^{2}$. The subtraction of the two terms leads to the integrability, and therefore it is not easy to find the proper domain of $\mathcal{E}$. Recently, Blatt [1] showed that $\mathcal{E}(\boldsymbol{f})<\infty$

2010 Mathematics Subject Classification. 53A04 (primary), 57M25, 57M27 (secondary).

Key words and phrases. Möbius energy, decomposition of energy, Möbius invariance.

${ }^{1}$ The second author is partly supported by Grant-in-Aid for scientific Research (A) (No. 22244010-01), Japan Society for the Promotion of Science.

Received April 1, 2014. 
if and only if $\boldsymbol{f} \in X=H^{1, \infty}(\mathbf{R} / \mathcal{L} \mathbf{Z}) \cap H^{3 / 2}(\mathbf{R} / \mathcal{L Z})$ and it is bi-Lischitz. In this paper, we establish a decomposition of $\mathcal{E}$ as

$$
\mathcal{E}(\boldsymbol{f})=\mathcal{E}_{1}(\boldsymbol{f})+\mathcal{E}_{2}(\boldsymbol{f})+4,
$$

where

$$
\mathcal{E}_{i}(\boldsymbol{f})=\iint_{(\mathbf{R} / \mathcal{L Z})^{2}} \mathscr{M}_{i}(\boldsymbol{f}) d s_{1} d s_{2}
$$

and $\mathscr{M}_{i}$ is the energy density of $\mathcal{E}_{i}$ (see the forthcoming Theorem 2.1). The first part $\mathcal{E}_{1}$ is a positive definite functional whose form implies the proper domain of $\mathcal{E}$ is $X$. The second one, $\mathcal{E}_{2}$, has a determinant structure which characterizes the cancellation of the integrand. Indeed each part $\mathcal{E}_{i}$ can be defined as an integral functional on $X$, i.e., whose integrand is absolutely integrable for $f \in X$.

The energy $\mathcal{E}$ is called the Möbius energy since it is invariant under Möbius transformations; this fact was shown by Freedman-He-Wang [3]. In section 3 we give an alternative proof of this, and discuss the Möbius invariance of each $\mathcal{E}_{i}$ in $X$ (Theorems 3.1 and 3.2). After discussing our results with O'Hara, he informed us that our second energy $\mathcal{E}_{2}$ is the same as the O'Hara-Solanes energy $E_{\mathrm{OS}}$ up to multiplication by a constant. The energy $E_{\mathrm{OS}}$ was first defined in [8] and they established its Möbius invariance for sufficiently smooth $f$. Note that $C^{\infty}$ is not dense in $X$.

Using our decomposition theorem, we can obtain explicit expressions of variational formulae of $\mathcal{E}$ with applicable estimates, which we will present in a forthcoming paper.

Acknowledgment. The authors would like to express their gratitude to Professor Jun O'Hara and Professor Philipp Reiter for discussions on their results.

\section{The decomposition theorem}

At a point where $f$ is differentiable, we denote the unit tangent vector by $\boldsymbol{\tau}=\boldsymbol{f}^{\prime}$. Similarly $\boldsymbol{\kappa}=\boldsymbol{\tau}^{\prime}$ stands for the curvature vector at a point where $\boldsymbol{f}$ is twice differentiable.

THEOREM 2.1. Let $\boldsymbol{f} \in X$ and suppose that there exists a positive constant $\lambda$ such that $\left\|\boldsymbol{f}\left(s_{1}\right)-\boldsymbol{f}\left(s_{2}\right)\right\|_{\mathbf{R}^{n}} \geq \lambda^{-1} \mathscr{D}\left(\boldsymbol{f}\left(s_{1}\right), \boldsymbol{f}\left(s_{2}\right)\right)$. The energy $\mathcal{E}(\boldsymbol{f})$ may be decomposed as

$$
\mathcal{E}(\boldsymbol{f})=\iint_{(\mathbf{R} / \mathcal{L} \mathbf{Z})^{2}}\left\{\mathscr{M}_{1}(\boldsymbol{f})\left(s_{1}, s_{2}\right)+\mathscr{M}_{2}(\boldsymbol{f})\left(s_{1}, s_{2}\right)\right\} d s_{1} d s_{2}+4
$$

where

$$
\mathscr{M}_{1}(\boldsymbol{f})=\frac{\left\|\boldsymbol{\tau}\left(s_{1}\right)-\boldsymbol{\tau}\left(s_{2}\right)\right\|_{\mathbf{R}^{n}}^{2}}{2\left\|\boldsymbol{f}\left(s_{1}\right)-\boldsymbol{f}\left(s_{2}\right)\right\|_{\mathbf{R}^{n}}^{2}}
$$


and

$$
\mathscr{M}_{2}(\boldsymbol{f})=\frac{2}{\left\|\boldsymbol{f}\left(s_{1}\right)-\boldsymbol{f}\left(s_{2}\right)\right\|_{\mathbf{R}^{n}}^{4}} \operatorname{det}\left(\begin{array}{cc}
\boldsymbol{\tau}\left(s_{1}\right) \cdot \boldsymbol{\tau}\left(s_{2}\right) & \left(\boldsymbol{f}\left(s_{1}\right)-\boldsymbol{f}\left(s_{2}\right)\right) \cdot \boldsymbol{\tau}\left(s_{1}\right) \\
\left(\boldsymbol{f}\left(s_{1}\right)-\boldsymbol{f}\left(s_{2}\right)\right) \cdot \boldsymbol{\tau}\left(s_{2}\right) & \left\|\boldsymbol{f}\left(s_{1}\right)-\boldsymbol{f}\left(s_{2}\right)\right\|_{\mathbf{R}^{n}}^{2}
\end{array}\right) .
$$

Moreover, each $\mathscr{M}_{i}(\boldsymbol{f})$ is absolutely integrable.

Proof. As we said in the Introduction, to deform the energy density, we first consider the integration in the sense of Cauchy's principal value, and show the absolute integrability later. We differentiate

$$
\mathscr{D}\left(\boldsymbol{f}\left(s_{1}\right), \boldsymbol{f}\left(s_{2}\right)\right)^{2}= \begin{cases}\left(s_{1}-s_{2}\right)^{2} & \left(s_{1} \leq s_{2} \leq s_{1}+\frac{\mathcal{L}}{2}(\bmod \mathcal{L})\right) \\ \left(s_{1}-s_{2}+\mathcal{L}\right)^{2} & \left(s_{1}+\frac{\mathcal{L}}{2} \leq s_{2} \leq s_{1}+\mathcal{L}(\bmod \mathcal{L})\right)\end{cases}
$$

with respect to $s_{2}$. In the sense of distributions,

$$
\frac{d}{d x} \log |x|=\text { p.v. } \frac{1}{x}
$$

and therefore

$$
\frac{\partial}{\partial s_{2}} \log \mathscr{D}\left(\boldsymbol{f}\left(s_{1}\right), \boldsymbol{f}\left(s_{2}\right)\right)= \begin{cases}- \text { p.v. } \frac{1}{s_{1}-s_{2}} \quad & \left(s_{1} \leq s_{2}<s_{1}+\frac{\mathcal{L}}{2}(\bmod \mathcal{L})\right) \\ - \text { p.v. } \frac{1}{s_{1}-s_{2}+\mathcal{L}} & \left(s_{1}+\frac{\mathcal{L}}{2}<s_{2} \leq s_{1}+\mathcal{L}(\bmod \mathcal{L})\right)\end{cases}
$$

Here, the distribution p.v. $\frac{1}{x}$ is given by

$$
\left\langle\text { p.v. } \frac{1}{x}, \varphi\right\rangle=\lim _{\varepsilon \rightarrow+0} \int_{|x| \geq \varepsilon} \frac{\varphi(x)}{x} d x
$$

for $\varphi \in C_{0}^{\infty}(\mathbf{R})$ (see [6]). Using the periodicity of $\mathscr{D}\left(\boldsymbol{f}\left(s_{1}\right), \boldsymbol{f}\left(s_{2}\right)\right)$, this equals

$$
\frac{\partial}{\partial s_{2}} \log \mathscr{D}\left(\boldsymbol{f}\left(s_{1}\right), \boldsymbol{f}\left(s_{2}\right)\right)= \begin{cases}- \text { p.v. } \frac{1}{s_{1}-s_{2}-\mathcal{L}} & \left(s_{2}+\frac{\mathcal{L}}{2}<s_{1} \leq s_{2}+\mathcal{L}(\bmod \mathcal{L})\right), \\ - \text { p.v. } \frac{1}{s_{1}-s_{2}} \quad\left(s_{2} \leq s_{1}<s_{2}+\frac{\mathcal{L}}{2}(\bmod \mathcal{L})\right) .\end{cases}
$$

Regarding this as a distribution of $s_{1}$, it is differentiable for $s_{1} \neq s_{2}+\mathcal{L} / 2$ in the weak sense and we obtain 


$$
\begin{aligned}
& \frac{\partial^{2}}{\partial s_{1} \partial s_{2}} \log \mathscr{D}\left(\boldsymbol{f}\left(s_{1}\right), \boldsymbol{f}\left(s_{2}\right)\right) \\
& \quad= \begin{cases}\text { p.v. } \frac{1}{\left(s_{1}-s_{2}-\mathcal{L}\right)^{2}} & \left(s_{2}+\frac{\mathcal{L}}{2}<s_{1} \leq s_{2}+\mathcal{L}(\bmod \mathcal{L})\right), \\
\text { p.v. } \frac{1}{\left(s_{1}-s_{2}\right)^{2}} & \left(s_{2} \leq s_{1}<s_{2}+\frac{\mathcal{L}}{2}(\bmod \mathcal{L})\right)\end{cases} \\
& =\text { p.v. } \frac{1}{\mathscr{D}\left(\boldsymbol{f}\left(s_{1}\right), \boldsymbol{f}\left(s_{2}\right)\right)^{2}} .
\end{aligned}
$$

As a function of $s_{2}, \frac{\partial}{\partial s_{2}} \log \mathscr{D}\left(\boldsymbol{f}\left(s_{1}\right), \boldsymbol{f}\left(s_{2}\right)\right)$ has a jump discontinuity at $s_{2}=$ $s_{1}+\mathcal{L} / 2$ with gap $-4 / \mathcal{L}$; that is,

$$
\lim _{s_{2} \rightarrow s_{1}+\mathcal{L} / 2+0} \frac{\partial}{\partial s_{2}} \log \mathscr{D}\left(\boldsymbol{f}\left(s_{1}\right), \boldsymbol{f}\left(s_{2}\right)\right)-\lim _{s_{2} \rightarrow s_{1}+\mathcal{L} / 2-0} \frac{\partial}{\partial s_{2}} \log \mathscr{D}\left(\boldsymbol{f}\left(s_{1}\right), \boldsymbol{f}\left(s_{2}\right)\right)=-\frac{4}{\mathcal{L}} .
$$

As functions of $s_{2}, \frac{\partial^{2}}{\partial s_{1} \partial s_{2}} \log \left\|\boldsymbol{f}\left(s_{1}\right)-\boldsymbol{f}\left(s_{2}\right)\right\|_{\mathbf{R}^{n}}$ is bounded at $s_{2}=s_{1} \pm \frac{\mathcal{L}}{2}$, and $\frac{\partial}{\partial s_{1}} \log \left\|\boldsymbol{f}\left(s_{1}\right)-\boldsymbol{f}\left(s_{2}\right)\right\|_{\mathbf{R}^{n}}$ is continuous at the same points. Therefore we get

$$
\begin{aligned}
& \int_{\varepsilon<\left|s_{1}-s_{2}\right| \leq \mathcal{L} / 2} \frac{1}{\mathscr{D}\left(\boldsymbol{f}\left(s_{1}\right), \boldsymbol{f}\left(s_{2}\right)\right)^{2}} d s_{2} \\
&=\lim _{\delta \rightarrow+0} \int_{\varepsilon<\left|s_{1}-s_{2}\right| \leq \mathcal{L} / 2-\delta} \frac{1}{\mathscr{D}\left(\boldsymbol{f}\left(s_{1}\right), \boldsymbol{f}\left(s_{2}\right)\right)^{2}} d s_{2} \\
&=\lim _{\delta \rightarrow+0} \int_{\varepsilon<\left|s_{1}-s_{2}\right| \leq \mathcal{L} / 2-\delta} \frac{\partial^{2}}{\partial s_{1} \partial s_{2}} \log \mathscr{D}\left(\boldsymbol{f}\left(s_{1}\right), \boldsymbol{f}\left(s_{2}\right)\right) d s_{2} \\
&=\lim _{\delta \rightarrow+0} \int_{\varepsilon<\left|s_{1}-s_{2}\right| \leq \mathcal{L} / 2-\delta} \frac{\partial^{2}}{\partial s_{1} \partial s_{2}} \\
& \quad \times\left(\log \left\|\boldsymbol{f}\left(s_{1}\right)-\boldsymbol{f}\left(s_{2}\right)\right\|_{\mathbf{R}^{n}}-\log \frac{\left\|\boldsymbol{f}\left(s_{1}\right)-\boldsymbol{f}\left(s_{2}\right)\right\|_{\mathbf{R}^{n}}}{\mathscr{D}\left(\boldsymbol{f}\left(s_{1}\right), \boldsymbol{f}\left(s_{2}\right)\right)}\right) d s_{2} \\
&=\int_{\varepsilon<\left|s_{1}-s_{2}\right| \leq \mathcal{L} / 2} \frac{\partial^{2}}{\partial s_{1} \partial s_{2}} \log \left\|\boldsymbol{f}\left(s_{1}\right)-\boldsymbol{f}\left(s_{2}\right)\right\|_{\mathbf{R}^{n}} d s_{2} \\
&-\lim _{\delta \rightarrow+0}\left[\frac{\partial}{\partial s_{1}} \log \frac{\left\|\boldsymbol{f}\left(s_{1}\right)-\boldsymbol{f}\left(s_{2}\right)\right\|_{\mathbf{R}^{n}}}{\mathscr{D}\left(\boldsymbol{f}\left(s_{1}\right), \boldsymbol{f}\left(s_{2}\right)\right)}\right]_{s_{2}=s_{1}-\mathcal{L} / 2+\delta}^{s_{2}=s_{1}-\varepsilon} \\
&-\lim _{\delta \rightarrow+0}\left[\frac{\partial}{\partial s_{1}} \log \frac{\left\|\boldsymbol{f}\left(s_{1}\right)-\boldsymbol{f}\left(s_{2}\right)\right\|_{\mathbf{R}^{n}}}{\mathscr{D}\left(\boldsymbol{f}\left(s_{1}\right), \boldsymbol{f}\left(s_{2}\right)\right)}\right]_{s_{2}=s_{1}+\varepsilon}^{s_{2}=s_{1}+\mathcal{L} / 2-\delta}
\end{aligned}
$$




$$
\begin{gathered}
=\int_{\varepsilon<\left|s_{1}-s_{2}\right| \leq \mathcal{L} / 2} \frac{\partial^{2}}{\partial s_{1} \partial s_{2}} \log \left\|\boldsymbol{f}\left(s_{1}\right)-\boldsymbol{f}\left(s_{2}\right)\right\|_{\mathbf{R}^{n}} d s_{2} \\
+\left[\frac{\partial}{\partial s_{1}} \log \frac{\left\|\boldsymbol{f}\left(s_{1}\right)-\boldsymbol{f}\left(s_{2}\right)\right\|_{\mathbf{R}^{n}}}{\mathscr{D}\left(\boldsymbol{f}\left(s_{1}\right), \boldsymbol{f}\left(s_{2}\right)\right)}\right]_{s_{2}=s_{1}-\varepsilon}^{s_{2}=s_{1}+\varepsilon}-\frac{4}{\mathcal{L}} .
\end{gathered}
$$

We integrate this with respect to $s_{1}$ and firstly note that

$$
\begin{aligned}
\int_{\mathbf{R} / \mathcal{L} \mathbf{Z}} & \left.\frac{\partial}{\partial s_{1}} \log \frac{\left\|\boldsymbol{f}\left(s_{1}\right)-\boldsymbol{f}\left(s_{2}\right)\right\|_{\mathbf{R}^{n}}}{\mathscr{D}\left(\boldsymbol{f}\left(s_{1}\right), \boldsymbol{f}\left(s_{2}\right)\right)}\right]_{s_{2}=s_{1}-\varepsilon}^{s_{2}=s_{1}+\varepsilon} d s_{1} \\
= & \int_{\mathbf{R} / \mathcal{L} \mathbf{Z}}\left[\frac{\left(\boldsymbol{f}\left(s_{1}\right)-\boldsymbol{f}\left(s_{2}\right)\right) \cdot \boldsymbol{\tau}\left(s_{1}\right)}{\left\|\boldsymbol{f}\left(s_{1}\right)-\boldsymbol{f}\left(s_{2}\right)\right\|_{\mathbf{R}^{n}}^{2}}-\frac{1}{s_{1}-s_{2}}\right]_{s_{2}=s_{1}-\varepsilon}^{s_{2}=s_{1}+\varepsilon} d_{1} \\
= & \int_{\mathbf{R} / \mathcal{L} \mathbf{Z}}\left\{\frac{\left(\boldsymbol{f}\left(s_{1}\right)-\boldsymbol{f}\left(s_{1}+\varepsilon\right)\right) \cdot \boldsymbol{\tau}\left(s_{1}\right)}{\left\|\boldsymbol{f}\left(s_{1}\right)-\boldsymbol{f}\left(s_{1}+\varepsilon\right)\right\|_{\mathbf{R}^{n}}^{2}}-\frac{\left(\boldsymbol{f}\left(s_{1}\right)-\boldsymbol{f}\left(s_{1}-\varepsilon\right)\right) \cdot \boldsymbol{\tau}\left(s_{1}\right)}{\left\|\boldsymbol{f}\left(s_{1}\right)-\boldsymbol{f}\left(s_{1}-\varepsilon\right)\right\|_{\mathbf{R}^{n}}^{2}}+\frac{2}{\varepsilon}\right\} d s_{1} \\
= & \int_{\mathbf{R} / \mathcal{L} \mathbf{Z}}\left\{\frac{\left(\boldsymbol{f}\left(s_{1}\right)-\boldsymbol{f}\left(s_{1}+\varepsilon\right)\right) \cdot \boldsymbol{\tau}\left(s_{1}\right)}{\left\|\boldsymbol{f}\left(s_{1}\right)-\boldsymbol{f}\left(s_{1}+\varepsilon\right)\right\|_{\mathbf{R}^{n}}^{2}}-\frac{\left(\boldsymbol{f}\left(s_{1}+\varepsilon\right)-\boldsymbol{f}\left(s_{1}\right)\right) \cdot \boldsymbol{\tau}\left(s_{1}+\varepsilon\right)}{\left\|\boldsymbol{f}\left(s_{1}+\varepsilon\right)-\boldsymbol{f}\left(s_{1}\right)\right\|_{\mathbf{R}^{n}}^{2}}+\frac{2}{\varepsilon}\right\} d s_{1} \\
= & \int_{\mathbf{R} / \mathcal{L} \mathbf{Z}}\left\{-\frac{\partial}{\partial s_{1}} \log \left\|\boldsymbol{f}\left(s_{1}\right)-\boldsymbol{f}\left(s_{1}+\varepsilon\right)\right\|_{\mathbf{R}^{n}}+\frac{2\left(\boldsymbol{f}\left(s_{1}+\varepsilon\right)-\boldsymbol{f}\left(s_{1}\right)\right) \cdot \tau\left(s_{1}\right)}{\left\|\boldsymbol{f}\left(s_{1}+\varepsilon\right)-\boldsymbol{f}\left(s_{1}\right)\right\|_{\mathbf{R}^{n}}^{2}}+\frac{2}{\varepsilon}\right\} d s_{1} \\
= & \int_{\mathbf{R} / \mathcal{L} \mathbf{Z}}\left[-2 \varepsilon\left(\frac{1}{\left\|\boldsymbol{f}\left(s_{1}+\varepsilon\right)-\boldsymbol{f}\left(s_{1}\right)\right\|_{\mathbf{R}^{n}}^{2}}-\frac{1}{\varepsilon^{2}}\right)\right. \\
& \left.\quad+\frac{2}{\left\|\boldsymbol{f}\left(s_{1}+\varepsilon\right)-\boldsymbol{f}\left(s_{1}\right)\right\|_{\mathbf{R}^{n}}^{2}} \int_{s_{1}}^{s_{1}+\varepsilon}\left(1-\boldsymbol{\tau}\left(s_{3}\right) \cdot \boldsymbol{\tau}\left(s_{1}\right)\right) d s_{3}\right] d s_{1} .
\end{aligned}
$$

We have

$$
\begin{aligned}
& \left|2 \varepsilon\left(\frac{1}{\left\|\boldsymbol{f}\left(s_{1}+\varepsilon\right)-\boldsymbol{f}\left(s_{1}\right)\right\|_{\mathbf{R}^{n}}^{2}}-\frac{1}{\varepsilon^{2}}\right)\right| \\
& \quad=\frac{2 \varepsilon}{\varepsilon^{2}\left\|\boldsymbol{f}\left(s_{1}+\varepsilon\right)-\boldsymbol{f}\left(s_{1}\right)\right\|_{\mathbf{R}^{n}}^{2}} \int_{s_{1}}^{s_{1}+\varepsilon} \int_{s_{1}}^{s_{1}+\varepsilon}\left(1-\boldsymbol{\tau}\left(s_{3}\right) \cdot \boldsymbol{\tau}\left(s_{4}\right)\right) d s_{3} d s_{4} \\
& \quad=\frac{1}{\varepsilon\left\|\boldsymbol{f}\left(s_{1}+\varepsilon\right)-\boldsymbol{f}\left(s_{1}\right)\right\|_{\mathbf{R}^{n}}^{2}} \int_{s_{1}}^{s_{1}+\varepsilon} \int_{s_{1}}^{s_{1}+\varepsilon}\left\|\boldsymbol{\tau}\left(s_{3}\right)-\boldsymbol{\tau}\left(s_{4}\right)\right\|_{\mathbf{R}^{n}}^{2} d s_{3} d s_{4}
\end{aligned}
$$

and using estimate $\left\|\boldsymbol{f}\left(s_{1}+\varepsilon\right)-\boldsymbol{f}\left(s_{1}\right)\right\|_{\mathbf{R}^{n}} \geq \lambda^{-1} \varepsilon$, along with a change in the order of integration, we obtain 


$$
\begin{aligned}
\int_{\mathbf{R} / \mathcal{L} \mathbf{Z}} & \frac{1}{\varepsilon\left\|\boldsymbol{f}\left(s_{1}+\varepsilon\right)-\boldsymbol{f}\left(s_{1}\right)\right\|_{\mathbf{R}^{n}}^{2}} \int_{s_{1}}^{s_{1}+\varepsilon} \int_{s_{1}}^{s_{1}+\varepsilon}\left\|\boldsymbol{\tau}\left(s_{3}\right)-\boldsymbol{\tau}\left(s_{4}\right)\right\|_{\mathbf{R}^{n}}^{2} d s_{3} d s_{4} d s_{1} \\
\leq & \frac{\lambda^{2}}{\varepsilon^{3}} \int_{\mathbf{R} / \mathcal{L} \mathbf{Z}} \int_{s_{1}}^{s_{1}+\varepsilon} \int_{s_{1}}^{s_{1}+\varepsilon}\left\|\boldsymbol{\tau}\left(s_{3}\right)-\boldsymbol{\tau}\left(s_{4}\right)\right\|_{\mathbf{R}^{n}}^{2} d s_{3} d s_{4} d s_{1} \\
\leq & \frac{\lambda^{2}}{\varepsilon^{3}} \int_{\mathbf{R} / \mathcal{L} \mathbf{Z}} \int_{s_{4}-\varepsilon}^{s_{4}+\varepsilon} \int_{s_{3}-\varepsilon}^{s_{3}}\left\|\boldsymbol{\tau}\left(s_{3}\right)-\boldsymbol{\tau}\left(s_{4}\right)\right\|_{\mathbf{R}^{n}}^{2} d s_{1} d s_{3} d s_{4} \\
\quad & \frac{\lambda^{2}}{\varepsilon^{2}} \int_{\mathbf{R} / \mathcal{L} \mathbf{Z}} \int_{s_{4}-\varepsilon}^{s_{4}+\varepsilon}\left\|\boldsymbol{\tau}\left(s_{3}\right)-\boldsymbol{\tau}\left(s_{4}\right)\right\|_{\mathbf{R}^{n}}^{2} d s_{3} d s_{4} \\
& \leq \lambda^{2} \int_{\mathbf{R} / \mathcal{L} \mathbf{Z}} \int_{s_{4}-\varepsilon}^{s_{4}+\varepsilon} \frac{\left\|\boldsymbol{\tau}\left(s_{3}\right)-\boldsymbol{\tau}\left(s_{4}\right)\right\|_{\mathbf{R}^{n}}^{2}}{\mathscr{D}\left(\boldsymbol{f}\left(s_{3}\right), \boldsymbol{f}\left(s_{4}\right)\right)^{2}} d s_{3} d s_{4} .
\end{aligned}
$$

Since

$$
\left[\boldsymbol{f}^{\prime}\right]_{H^{1 / 2}}^{2}=\iint_{(\mathbf{R} / \mathcal{L Z})^{2}} \frac{\left\|\boldsymbol{\tau}\left(s_{3}\right)-\boldsymbol{\tau}\left(s_{4}\right)\right\|_{\mathbf{R}^{n}}^{2}}{\mathscr{D}\left(\boldsymbol{f}\left(s_{3}\right), \boldsymbol{f}\left(s_{4}\right)\right)^{2}} d s_{3} d s_{4}
$$

is finite, the absolute continuity of integration yields

$$
\lim _{\varepsilon \rightarrow+0} \int_{\mathbf{R} / \mathcal{L} \mathbf{Z}} \frac{1}{\varepsilon\left\|\boldsymbol{f}\left(s_{1}+\varepsilon\right)-\boldsymbol{f}\left(s_{1}\right)\right\|_{\mathbf{R}^{n}}^{2}} \int_{s_{1}}^{s_{1}+\varepsilon} \int_{s_{1}}^{s_{1}+\varepsilon}\left\|\boldsymbol{\tau}\left(s_{3}\right)-\boldsymbol{\tau}\left(s_{4}\right)\right\|_{\mathbf{R}^{n}}^{2} d s_{3} d s_{4} d s_{1}=0 .
$$

Similarly we have

$$
\begin{aligned}
& \mid \int_{\mathbf{R} / \mathcal{L} \mathbf{Z}} \frac{2}{\left\|\boldsymbol{f}\left(s_{1}+\varepsilon\right)-\boldsymbol{f}\left(s_{1}\right)\right\|_{\mathbf{R}^{n}}^{2}} \int_{s_{1}}^{s_{1}+\varepsilon}\left(1-\boldsymbol{\tau}\left(s_{3}\right) \cdot \boldsymbol{\tau}\left(s_{1}\right)\right) d s_{3} d s_{1} \mid \\
& \quad= \int_{\mathbf{R} / \mathcal{L} \mathbf{Z}} \frac{1}{\left\|\boldsymbol{f}\left(s_{1}+\varepsilon\right)-\boldsymbol{f}\left(s_{1}\right)\right\|_{\mathbf{R}^{n}}^{2}} \int_{s_{1}}^{s_{1}+\varepsilon}\left\|\boldsymbol{\tau}\left(s_{3}\right)-\boldsymbol{\tau}\left(s_{1}\right)\right\|_{\mathbf{R}^{n}}^{2} d s_{3} d s_{1} \\
& \quad \leq \lambda^{2} \int_{\mathbf{R} / \mathcal{L} \mathbf{Z}} \int_{s_{1}}^{s_{1}+\varepsilon} \frac{\left\|\boldsymbol{\tau}\left(s_{3}\right)-\boldsymbol{\tau}\left(s_{1}\right)\right\|_{\mathbf{R}^{n}}^{2}}{\mathscr{D}\left(\boldsymbol{f}\left(s_{3}\right), \boldsymbol{f}\left(s_{1}\right)\right)^{2}} d s_{3} d s_{1} \rightarrow 0 \quad \text { as } \varepsilon \rightarrow+0 .
\end{aligned}
$$

Hence we obtain

$$
\int_{\mathbf{R} / \mathcal{L} \mathbf{Z}}\left[\frac{\partial}{\partial s_{1}} \log \frac{\left\|\boldsymbol{f}\left(s_{1}\right)-\boldsymbol{f}\left(s_{2}\right)\right\|_{\mathbf{R}^{n}}}{\mathscr{D}\left(\boldsymbol{f}\left(s_{1}\right), \boldsymbol{f}\left(s_{2}\right)\right)}\right]_{s_{2}=s_{1}-\varepsilon}^{s_{2}=s_{1}+\varepsilon} d s_{1} \rightarrow 0 \quad \text { as } \varepsilon \rightarrow+0
$$

which leads to the expression $\mathcal{E}(\boldsymbol{f})=$ p.v. $\iint_{(\mathbf{R} / \mathcal{L} \mathbf{Z})^{2}}\left(\frac{1}{\left\|\boldsymbol{f}\left(s_{1}\right)-\boldsymbol{f}\left(s_{2}\right)\right\|_{\mathbf{R}^{n}}^{2}}-\frac{\partial^{2}}{\partial s_{1} \partial s_{2}} \log \left\|\boldsymbol{f}\left(s_{1}\right)-\boldsymbol{f}\left(s_{2}\right)\right\|_{\mathbf{R}^{n}}\right) d s_{1} d s_{2}+4$.

Manipulating the above log term, we obtain 
(2) $\mathcal{E}(\boldsymbol{f})-4=$ p.v. $\iint_{(\mathbf{R} / \mathcal{L} \mathbf{Z})^{2}}\left\{\frac{1+\boldsymbol{\tau}\left(s_{1}\right) \cdot \boldsymbol{\tau}\left(s_{2}\right)}{\left\|\boldsymbol{f}\left(s_{1}\right)-\boldsymbol{f}\left(s_{2}\right)\right\|_{\mathbf{R}^{n}}^{2}}\right.$

$$
\left.-\frac{2\left\{\left(\boldsymbol{f}\left(s_{1}\right)-\boldsymbol{f}\left(s_{2}\right)\right) \cdot \boldsymbol{\tau}\left(s_{1}\right)\right\}\left\{\left(\boldsymbol{f}\left(s_{1}\right)-\boldsymbol{f}\left(s_{2}\right)\right) \cdot \boldsymbol{\tau}\left(s_{2}\right)\right\}}{\left\|\boldsymbol{f}\left(s_{1}\right)-\boldsymbol{f}\left(s_{2}\right)\right\|_{\mathbf{R}^{n}}^{4}}\right\} d s_{1} d s_{2} .
$$

The density of this integral is expressed as

$$
\begin{aligned}
\frac{1+}{\left\|\boldsymbol{f}\left(s_{1}\right)-\boldsymbol{f}\left(s_{2}\right)\right\|_{\mathbf{R}^{n}}^{2}}-\frac{2\left\{\left(\boldsymbol{f}\left(s_{1}\right)-\boldsymbol{f}\left(s_{2}\right)\right) \cdot \boldsymbol{\tau}\left(s_{1}\right)\right\}\left\{\left(\boldsymbol{f}\left(s_{1}\right)-\boldsymbol{f}\left(s_{2}\right)\right) \cdot \boldsymbol{\tau}\left(s_{2}\right)\right\}}{\left\|\boldsymbol{f}\left(s_{1}\right)-\boldsymbol{f}\left(s_{2}\right)\right\|_{\mathbf{R}^{n}}^{4}} & \\
= & \frac{1-\boldsymbol{\tau}\left(s_{1}\right) \cdot \boldsymbol{\tau}\left(s_{2}\right)}{\left\|\boldsymbol{f}\left(s_{1}\right)-\boldsymbol{f}\left(s_{2}\right)\right\|_{\mathbf{R}^{n}}^{2}}+\frac{2 \boldsymbol{\tau}\left(s_{1}\right) \cdot \boldsymbol{\tau}\left(s_{2}\right)\left\|\boldsymbol{f}\left(s_{1}\right)-\boldsymbol{f}\left(s_{2}\right)\right\|_{\mathbf{R}^{n}}^{2}}{\left\|\boldsymbol{f}\left(s_{1}\right)-\boldsymbol{f}\left(s_{2}\right)\right\|_{\mathbf{R}^{n}}^{4}} \\
& \quad-\frac{\left\{\left(\boldsymbol{f}\left(s_{1}\right)-\boldsymbol{f}\left(s_{2}\right)\right) \cdot \boldsymbol{\tau}\left(s_{1}\right)\right\}\left\{\left(\boldsymbol{f}\left(s_{1}\right)-\boldsymbol{f}\left(s_{2}\right)\right) \cdot \boldsymbol{\tau}\left(s_{2}\right)\right\}}{\left\|\boldsymbol{f}\left(s_{1}\right)-\boldsymbol{f}\left(s_{2}\right)\right\|_{\mathbf{R}^{n}}^{4}} \\
= & \frac{\left\|\boldsymbol{\tau}\left(s_{1}\right)-\boldsymbol{\tau}\left(s_{2}\right)\right\|_{\mathbf{R}^{n}}^{2}}{2\left\|\boldsymbol{f}\left(s_{1}\right)-\boldsymbol{f}\left(s_{2}\right)\right\|_{\mathbf{R}^{n}}^{2}} \\
& +\frac{2}{\left\|\boldsymbol{f}\left(s_{1}\right)-\boldsymbol{f}\left(s_{2}\right)\right\|_{\mathbf{R}^{n}}^{4}} \operatorname{det}\left(\begin{array}{cc}
\boldsymbol{\tau}\left(s_{1}\right) \cdot \boldsymbol{\tau}\left(s_{2}\right) & \left.\left(\boldsymbol{f}\left(s_{1}\right)-\boldsymbol{f}\left(s_{2}\right)\right) \cdot \boldsymbol{\tau}\left(s_{2}\right)\right) \cdot \boldsymbol{\tau}\left(s_{1}\right) \\
= & \left\|\boldsymbol{f}\left(s_{1}\right)-\boldsymbol{f}\left(s_{2}\right)\right\|_{\mathbf{R}^{n}}^{2}
\end{array}\right) \\
\mathscr{M}_{1}(\boldsymbol{f})+\mathscr{M}_{2}(\boldsymbol{f}) &
\end{aligned}
$$

and it remains to remove p.v. in front of the double integral in (2). To this end it is enough to see

$$
\mathscr{M}_{1}(\boldsymbol{f})+\mathscr{M}_{2}(\boldsymbol{f}) \geq 0 .
$$

For a function $\boldsymbol{v}$ on $\mathbf{R} / \mathcal{L} \mathbf{Z}$, we define $R \boldsymbol{v}=R \boldsymbol{v}\left(s_{1}, s_{2}\right)$ by

$$
R \boldsymbol{v}=\frac{\left|s_{1}-s_{2}\right|\left(\boldsymbol{v}\left(s_{1}\right)-\boldsymbol{v}\left(s_{2}\right)\right)}{\left\|\boldsymbol{f}\left(s_{1}\right)-\boldsymbol{f}\left(s_{2}\right)\right\|_{\mathbf{R}^{n}}\left(s_{1}-s_{2}\right)}
$$

and observe that $R \boldsymbol{f}$ is clearly a unit vector field. In addition, we define

$$
\boldsymbol{v}_{*}=R \boldsymbol{f}-\left(R \boldsymbol{f} \cdot \boldsymbol{\tau}_{m}\right) \boldsymbol{\tau}_{m} .
$$

Here, the vector $\boldsymbol{\tau}_{m}=\boldsymbol{\tau}_{m}\left(s_{1}, s_{2}\right)$ is defined by

$$
\boldsymbol{\tau}_{m}=\frac{\boldsymbol{\tau}\left(s_{1}\right)+\boldsymbol{\tau}\left(s_{2}\right)}{\left\|\boldsymbol{\tau}\left(s_{1}\right)+\boldsymbol{\tau}\left(s_{2}\right)\right\|_{\mathbf{R}^{n}}}
$$

if $\boldsymbol{\tau}\left(s_{1}\right)+\boldsymbol{\tau}\left(s_{2}\right) \neq \boldsymbol{o}$, and otherwise $\boldsymbol{\tau}_{m}$ is defined to be any unit vector satisfying $\tau_{m} \cdot \tau\left(s_{1}\right)=0$. First, we consider the non-negativity of the numerator of the right-hand side of

$$
\mathscr{M}_{1}(\boldsymbol{f})\left(s_{1}, s_{2}\right)+\mathscr{M}_{2}(\boldsymbol{f})\left(s_{1}, s_{2}\right)=\frac{1+\boldsymbol{\tau}\left(s_{1}\right) \cdot \boldsymbol{\tau}\left(s_{2}\right)-2\left(R \boldsymbol{f} \cdot \boldsymbol{\tau}\left(s_{1}\right)\right)\left(R \boldsymbol{f} \cdot \boldsymbol{\tau}\left(s_{2}\right)\right)}{\left\|\boldsymbol{f}\left(s_{1}\right)-\boldsymbol{f}\left(s_{2}\right)\right\|_{\mathbf{R}^{n}}^{2}} .
$$


Note that we have

$$
\boldsymbol{\tau}_{m} \cdot \boldsymbol{\tau}\left(s_{1}\right)=\boldsymbol{\tau}_{m} \cdot \boldsymbol{\tau}\left(s_{2}\right)=\frac{1}{2}\left\|\boldsymbol{\tau}\left(s_{1}\right)+\boldsymbol{\tau}\left(s_{2}\right)\right\|_{\mathbf{R}^{n}} .
$$

Since $\boldsymbol{v}_{*} \perp\left(\boldsymbol{\tau}\left(s_{1}\right)+\boldsymbol{\tau}\left(s_{2}\right)\right)$, we have

$$
\boldsymbol{v}_{*} \cdot\left(\boldsymbol{\tau}\left(s_{1}\right)+\boldsymbol{\tau}\left(s_{2}\right)\right)=0
$$

and squaring both sides, we have

$$
\left(\boldsymbol{v}_{*} \cdot \boldsymbol{\tau}\left(s_{1}\right)\right)^{2}+2\left(\boldsymbol{v}_{*} \cdot \boldsymbol{\tau}\left(s_{1}\right)\right)\left(\boldsymbol{v}_{*} \cdot \boldsymbol{\tau}\left(s_{2}\right)\right)+\left(\boldsymbol{v}_{*} \cdot \boldsymbol{\tau}\left(s_{2}\right)\right)^{2}=0 .
$$

From (3), it follows that

$$
\begin{aligned}
R \boldsymbol{f} \cdot \boldsymbol{\tau}\left(s_{j}\right) & =\left(R \boldsymbol{f} \cdot \boldsymbol{\tau}_{m}\right)\left(\boldsymbol{\tau}_{m} \cdot \boldsymbol{\tau}\left(s_{j}\right)\right)+\boldsymbol{v}_{*} \cdot \boldsymbol{\tau}\left(s_{j}\right) \\
& =\frac{1}{2}\left\|\boldsymbol{\tau}\left(s_{1}\right)+\boldsymbol{\tau}\left(s_{2}\right)\right\|_{\mathbf{R}^{n}}\left(R \boldsymbol{f} \cdot \boldsymbol{\tau}_{m}\right)+\boldsymbol{v}_{*} \cdot \boldsymbol{\tau}\left(s_{j}\right) .
\end{aligned}
$$

Using this equation and (4)-(5), we get

$$
\begin{aligned}
\left(R \boldsymbol{f} \cdot \boldsymbol{\tau}\left(s_{1}\right)\right)\left(R \boldsymbol{f} \cdot \boldsymbol{\tau}\left(s_{2}\right)\right) \\
=\frac{1}{4}\left\|\boldsymbol{\tau}\left(s_{1}\right)+\boldsymbol{\tau}\left(s_{2}\right)\right\|_{\mathbf{R}^{n}}^{2}\left(R \boldsymbol{f} \cdot \boldsymbol{\tau}_{m}\right)^{2} \\
\quad+\frac{1}{2}\left\|\boldsymbol{\tau}\left(s_{1}\right)+\boldsymbol{\tau}\left(s_{2}\right)\right\|_{\mathbf{R}^{n}}\left(R \boldsymbol{f} \cdot \boldsymbol{\tau}_{m}\right)\left\{\boldsymbol{v}_{*} \cdot\left(\boldsymbol{\tau}\left(s_{1}\right)+\boldsymbol{\tau}\left(s_{2}\right)\right)\right\} \\
\quad+\left(\boldsymbol{v}_{*} \cdot \boldsymbol{\tau}\left(s_{1}\right)\right)\left(\boldsymbol{v}_{*} \cdot \boldsymbol{\tau}\left(s_{2}\right)\right) \\
=\frac{1}{4}\left\|\boldsymbol{\tau}\left(s_{1}\right)+\boldsymbol{\tau}\left(s_{2}\right)\right\|_{\mathbf{R}^{n}}^{2}\left(R \boldsymbol{f} \cdot \boldsymbol{\tau}_{m}\right)^{2}-\frac{1}{2}\left\{\left(\boldsymbol{v}_{*} \cdot \boldsymbol{\tau}\left(s_{1}\right)\right)^{2}+\left(\boldsymbol{v}_{*} \cdot \boldsymbol{\tau}\left(s_{2}\right)\right)^{2}\right\}
\end{aligned}
$$

and therefore

$$
\begin{aligned}
\| \boldsymbol{f}\left(s_{1}\right) & -\boldsymbol{f}\left(s_{2}\right) \|_{\mathbf{R}^{n}}^{2}\left(\mathscr{M}_{1}(\boldsymbol{f})\left(s_{1}, s_{2}\right)+\mathscr{M}_{2}(\boldsymbol{f})\left(s_{1}, s_{2}\right)\right) \\
= & \frac{1}{2}\left\|\boldsymbol{\tau}\left(s_{1}\right)+\boldsymbol{\tau}\left(s_{2}\right)\right\|_{\mathbf{R}^{n}}^{2}-\frac{1}{2}\left\|\boldsymbol{\tau}\left(s_{1}\right)+\boldsymbol{\tau}\left(s_{2}\right)\right\|_{\mathbf{R}^{n}}^{2}\left(R \boldsymbol{f} \cdot \boldsymbol{\tau}_{m}\right)^{2} \\
& +\left(\boldsymbol{v}_{*} \cdot \boldsymbol{\tau}\left(s_{1}\right)\right)^{2}+\left(\boldsymbol{v}_{*} \cdot \boldsymbol{\tau}\left(s_{2}\right)\right)^{2} \\
= & \frac{1}{2}\left\|\boldsymbol{\tau}\left(s_{1}\right)+\boldsymbol{\tau}\left(s_{2}\right)\right\|_{\mathbf{R}^{n}}^{2}\left\|\boldsymbol{v}_{*}\right\|_{\mathbf{R}^{n}}^{2}+\left(\boldsymbol{v}_{*} \cdot \boldsymbol{\tau}\left(s_{1}\right)\right)^{2}+\left(\boldsymbol{v}_{*} \cdot \boldsymbol{\tau}\left(s_{2}\right)\right)^{2} \geq 0
\end{aligned}
$$

as desired.

Finally we show the absolute integrability of each $\mathscr{M}_{i}(\boldsymbol{f})$. The integrand $\mathscr{M}_{1}(\boldsymbol{f})$ is non-negative and

$$
\begin{aligned}
\int_{(\mathbf{R} / \mathcal{L} \mathbf{Z})^{2}} \mathscr{M}_{1}(\boldsymbol{f}) d s_{1} d s_{2} & \leq \frac{\lambda^{2}}{2} \int_{(\mathbf{R} / \mathcal{L} \mathbf{Z})^{2}} \frac{\left\|\boldsymbol{\tau}\left(s_{1}\right)-\boldsymbol{\tau}\left(s_{2}\right)\right\|_{\mathbf{R}^{n}}^{2}}{\mathscr{D}\left(\boldsymbol{f}\left(s_{1}\right), \boldsymbol{f}\left(s_{2}\right)\right)^{2}} d s_{1} d s_{2} \\
& =\frac{\lambda^{2}}{2}\left[\boldsymbol{f}^{\prime}\right]_{H^{1 / 2}}^{2}<\infty
\end{aligned}
$$


which shows the absolute integrability of $\mathscr{M}_{1}(\boldsymbol{f})$. Both $\mathscr{M}_{1}(\boldsymbol{f})$ and $\mathscr{M}_{1}(\boldsymbol{f})+$ $\mathscr{M}_{2}(\boldsymbol{f})$ are absolutely integrable, hence so is $\mathscr{M}_{2}(\boldsymbol{f})$.

Remark 2.1. O'Hara kindly informed the authors that if $f$ is sufficiently smooth, then (2) can be shown by using the cosine formula [5].

\section{Möbius invariance}

In this section we discuss the invariance of $\mathscr{M}_{i}$ under Möbius transformations.

The invariance under dilation can be easily shown. In subsection 3.1, we show the invariance of the sum $\mathcal{E}_{1}+\mathcal{E}_{2}$ under the inversion

$$
\boldsymbol{f} \mapsto \boldsymbol{p}=\boldsymbol{c}+\frac{r^{2}(\boldsymbol{f}-\boldsymbol{c})}{\|\boldsymbol{f}-\boldsymbol{c}\|_{\mathbf{R}^{n}}^{2}}
$$

with respect to the sphere with center $c$ and radius $r$. Note that we assume neither the finiteness of energy $\mathcal{E}(\boldsymbol{f})<\infty$ nor $\boldsymbol{f}(\mathbf{R} / \mathcal{L} \mathbf{Z}) \ni \boldsymbol{c}$. Indeed we show the invariance of the sum of measures $\left(\mathscr{M}_{1}+\mathscr{M}_{2}\right) d s_{1} d s_{2}$. This fact was shown by Freedman-He-Wang [3] for $\boldsymbol{f}$ which are parameterized by arc-length, and here we present an alternative proof. We discuss the invariance of each $\mathcal{E}_{i}$ under the inversion assuming the finiteness of energy and $c \notin f(\mathbf{R} / \mathcal{L} \mathbf{Z})$ in subsection 3.2. For the case $c \notin \boldsymbol{f}(\mathbf{R} / \mathcal{L Z})$, see the final remark at the end of this section.

We now introduce the new symbol $\Delta$ which represents the difference between the function value for $s_{1}$ and that for $s_{2}$; that is,

$$
\Delta \boldsymbol{f}=\boldsymbol{f}\left(s_{1}\right)-\boldsymbol{f}\left(s_{2}\right), \quad \Delta \boldsymbol{\tau}=\boldsymbol{\tau}\left(s_{1}\right)-\boldsymbol{\tau}\left(s_{2}\right), \quad \Delta s=s_{1}-s_{2} .
$$

\subsection{The invariance of the sum of energies}

In this section we show the invariance of $\left(\mathscr{M}_{1}+\mathscr{M}_{2}\right) d s_{1} d s_{2}$ and as a consequence the invariance of the sum of energies follows. First we note

$$
\mathscr{M}_{1}(\boldsymbol{f})+\mathscr{M}_{2}(\boldsymbol{f})=\frac{1+\boldsymbol{\tau}\left(s_{1}\right) \cdot \boldsymbol{\tau}\left(s_{2}\right)}{\|\Delta \boldsymbol{f}\|_{\mathbf{R}^{n}}^{2}}-\frac{2\left(\Delta \boldsymbol{f} \cdot \boldsymbol{\tau}\left(s_{1}\right)\right)\left(\Delta \boldsymbol{f} \cdot \boldsymbol{\tau}\left(s_{2}\right)\right)}{\|\Delta \boldsymbol{f}\|_{\mathbf{R}^{n}}^{4}} .
$$

Even if $s$ is an arc-length parameter for $\boldsymbol{f}$, it is not necessarily so for $\boldsymbol{p}$. Therefore we use a general parameter $\theta$ instead of $s$, and the energy density with respect to $d \theta_{1} d \theta_{2}$ is

$$
\begin{aligned}
\left(\mathscr{M}_{1}(\boldsymbol{f})+\mathscr{M}_{2}(\boldsymbol{f})\right)\left\|\dot{\boldsymbol{f}}\left(\theta_{1}\right)\right\|_{\mathbf{R}^{n}}\left\|\dot{\boldsymbol{f}}\left(\theta_{2}\right)\right\|_{\mathbf{R}^{n}} \\
=\frac{\left\|\dot{\boldsymbol{f}}\left(\theta_{1}\right)\right\|_{\mathbf{R}^{n}}\left\|\dot{\boldsymbol{f}}\left(\theta_{2}\right)\right\|_{\mathbf{R}^{n}}+\dot{\boldsymbol{f}}\left(\theta_{1}\right) \cdot \dot{\boldsymbol{f}}\left(\theta_{2}\right)}{\left\|\boldsymbol{f}\left(\theta_{1}\right)-\boldsymbol{f}\left(\theta_{2}\right)\right\|_{\mathbf{R}^{n}}^{2}} \\
\quad+\frac{1}{2}\left(\frac{\partial}{\partial \theta_{1}} \log \left\|\boldsymbol{f}\left(\theta_{1}\right)-\boldsymbol{f}\left(\theta_{2}\right)\right\|_{\mathbf{R}^{n}}^{2}\right)\left(\frac{\partial}{\partial \theta_{2}} \log \left\|\boldsymbol{f}\left(\theta_{1}\right)-\boldsymbol{f}\left(\theta_{2}\right)\right\|_{\mathbf{R}^{n}}^{2}\right) .
\end{aligned}
$$

Here $\dot{f}$ means the differentiation of $\boldsymbol{f}$ with respect to the general parameter, and similarly for other functions. 
THEOREM 3.1. Let

$$
\boldsymbol{f} \mapsto \boldsymbol{p}=\boldsymbol{c}+\frac{r^{2}(\boldsymbol{f}-\boldsymbol{c})}{\|\boldsymbol{f}-\boldsymbol{c}\|_{\mathbf{R}^{n}}^{2}}
$$

be an inversion with respect to the sphere with center $c$ and radius $r$. Then,

$$
\begin{aligned}
& \left(\mathscr{M}_{1}(\boldsymbol{f})+\mathscr{M}_{2}(\boldsymbol{f})\right)\left\|\dot{\boldsymbol{f}}\left(\theta_{1}\right)\right\|_{\mathbf{R}^{n}}\left\|\dot{\boldsymbol{f}}\left(\theta_{2}\right)\right\|_{\mathbf{R}^{n}} \\
& \quad-\left(\mathscr{M}_{1}(\boldsymbol{p})+\mathscr{M}_{2}(\boldsymbol{p})\right)\left\|\dot{\boldsymbol{p}}\left(\theta_{1}\right)\right\|_{\mathbf{R}^{n}}\left\|\dot{\boldsymbol{p}}\left(\theta_{2}\right)\right\|_{\mathbf{R}^{n}}=0
\end{aligned}
$$

holds for $\theta_{1}$ and $\theta_{2}$ such that

$$
\boldsymbol{f}\left(\theta_{1}\right) \neq \boldsymbol{f}\left(\theta_{2}\right), \quad \boldsymbol{f}\left(\theta_{i}\right) \neq \boldsymbol{c} \quad(i=1,2) .
$$

Proof. We decompose the difference between the density for $f$ and that for $p$ as follows:

$$
\begin{aligned}
\left(\mathscr{M}_{1}(\boldsymbol{f})+\mathscr{M}_{2}(\boldsymbol{f})\right)\left\|\dot{\boldsymbol{f}}\left(\theta_{1}\right)\right\|_{\mathbf{R}^{n}}\left\|\dot{\boldsymbol{f}}\left(\theta_{2}\right)\right\|_{\mathbf{R}^{n}} & \\
& -\left(\mathscr{M}_{1}(\boldsymbol{p})+\mathscr{M}_{2}(\boldsymbol{p})\right)\left\|\dot{\boldsymbol{p}}\left(\theta_{1}\right)\right\|_{\mathbf{R}^{n}}\left\|\dot{\boldsymbol{p}}\left(\theta_{2}\right)\right\|_{\mathbf{R}^{n}} \\
= & J_{1}+J_{2}+J_{3},
\end{aligned}
$$

where

$$
\begin{aligned}
J_{1}= & \frac{\left\|\dot{\boldsymbol{f}}\left(\theta_{1}\right)\right\|_{\mathbf{R}^{n}}\left\|\dot{\boldsymbol{f}}\left(\theta_{2}\right)\right\|_{\mathbf{R}^{n}}}{\left\|\boldsymbol{f}\left(\theta_{1}\right)-\boldsymbol{f}\left(\theta_{2}\right)\right\|_{\mathbf{R}^{n}}^{2}}-\frac{\left\|\dot{\boldsymbol{p}}\left(\theta_{1}\right)\right\|_{\mathbf{R}^{n}}\left\|\dot{\boldsymbol{p}}\left(\theta_{2}\right)\right\|_{\mathbf{R}^{n}}}{\left\|\boldsymbol{p}\left(\theta_{1}\right)-\boldsymbol{p}\left(\theta_{2}\right)\right\|_{\mathbf{R}^{n}}^{2}}, \\
J_{2}= & \frac{\dot{\boldsymbol{f}}\left(\theta_{1}\right) \cdot \dot{\boldsymbol{f}}\left(\theta_{2}\right)}{\left\|\boldsymbol{f}\left(\theta_{1}\right)-\boldsymbol{f}\left(\theta_{2}\right)\right\|_{\mathbf{R}^{n}}^{2}}-\frac{\dot{\boldsymbol{p}}\left(\theta_{1}\right) \cdot \dot{\boldsymbol{p}}\left(\theta_{2}\right)}{\left\|\boldsymbol{p}\left(\theta_{1}\right)-\boldsymbol{p}\left(\theta_{2}\right)\right\|_{\mathbf{R}^{n}}^{2}}, \\
J_{3}= & \frac{1}{2}\left\{\left(\frac{\partial}{\partial \theta_{1}} \log \left\|\boldsymbol{f}\left(\theta_{1}\right)-\boldsymbol{f}\left(\theta_{2}\right)\right\|_{\mathbf{R}^{n}}^{2}\right)\left(\frac{\partial}{\partial \theta_{2}} \log \left\|\boldsymbol{f}\left(\theta_{1}\right)-\boldsymbol{f}\left(\theta_{2}\right)\right\|_{\mathbf{R}^{n}}^{2}\right)\right. \\
& \left.\quad-\left(\frac{\partial}{\partial \theta_{1}} \log \left\|\boldsymbol{p}\left(\theta_{1}\right)-\boldsymbol{p}\left(\theta_{2}\right)\right\|_{\mathbf{R}^{n}}^{2}\right)\left(\frac{\partial}{\partial \theta_{2}} \log \left\|\boldsymbol{p}\left(\theta_{1}\right)-\boldsymbol{p}\left(\theta_{2}\right)\right\|_{\mathbf{R}^{n}}^{2}\right)\right\} .
\end{aligned}
$$

It holds that

$$
\begin{aligned}
\| \boldsymbol{p}\left(\theta_{1}\right) & -\boldsymbol{p}\left(\theta_{2}\right) \|_{\mathbf{R}^{n}}^{2} \\
& =\left\|\frac{r^{2}\left(\boldsymbol{f}\left(\theta_{1}\right)-\boldsymbol{c}\right)}{\left\|\boldsymbol{f}\left(\theta_{1}\right)-\boldsymbol{c}\right\|_{\mathbf{R}^{n}}^{2}}-\frac{r^{2}\left(\boldsymbol{f}\left(\theta_{2}\right)-\boldsymbol{c}\right)}{\left\|\boldsymbol{f}\left(\theta_{2}\right)-\boldsymbol{c}\right\|_{\mathbf{R}^{n}}^{2}}\right\|_{\mathbf{R}^{n}}^{2} \\
& =r^{4}\left\{\frac{1}{\left\|\boldsymbol{f}\left(\theta_{1}\right)-\boldsymbol{c}\right\|_{\mathbf{R}^{n}}^{2}}-\frac{2\left(\boldsymbol{f}\left(\theta_{1}\right)-\boldsymbol{c}\right) \cdot\left(\boldsymbol{f}\left(\theta_{2}\right)-\boldsymbol{c}\right)}{\left\|\boldsymbol{f}\left(\theta_{1}\right)-\boldsymbol{c}\right\|_{\mathbf{R}^{n}}^{2}\left\|\boldsymbol{f}\left(\theta_{2}\right)-\boldsymbol{c}\right\|_{\mathbf{R}^{n}}^{2}}+\frac{1}{\left\|\boldsymbol{f}\left(\theta_{2}\right)-\boldsymbol{c}\right\|_{\mathbf{R}^{n}}^{2}}\right\} \\
& =\frac{r^{4}\left\|\boldsymbol{f}\left(\theta_{1}\right)-\boldsymbol{f}\left(\theta_{2}\right)\right\|_{\mathbf{R}^{n}}^{2}}{\left\|\boldsymbol{f}\left(\theta_{1}\right)-\boldsymbol{c}\right\|_{\mathbf{R}^{n}}^{2}\left\|\boldsymbol{f}\left(\theta_{2}\right)-\boldsymbol{c}\right\|_{\mathbf{R}^{n}}^{2}} .
\end{aligned}
$$


If we define the projection $P_{c}(\theta)$ and $P_{c}^{\perp}(\theta)$ for a vector $\boldsymbol{v}$ by

$$
P_{c}(\theta) \boldsymbol{v}=\left(\boldsymbol{v} \cdot \frac{\boldsymbol{f}(\theta)-\boldsymbol{c}}{\|\boldsymbol{f}(\theta)-\boldsymbol{c}\|_{\mathbf{R}^{n}}}\right) \frac{\boldsymbol{f}(\theta)-\boldsymbol{c}}{\|\boldsymbol{f}(\theta)-\boldsymbol{c}\|_{\mathbf{R}^{n}}}, \quad P_{c}^{\perp}(\theta)=I-P_{c}(\theta),
$$

then the derivative of $\boldsymbol{p}(\theta)$ may be expressed as

$$
\begin{aligned}
\dot{\boldsymbol{p}}(\theta) & =r^{2}\left[\frac{\dot{\boldsymbol{f}}(\theta)}{\|\boldsymbol{f}(\theta)-\boldsymbol{c}\|_{\mathbf{R}^{n}}^{2}}-\frac{2\{\dot{\boldsymbol{f}}(\theta) \cdot(\boldsymbol{f}(\theta)-\boldsymbol{c})\}(\boldsymbol{f}(\theta)-\boldsymbol{c})}{\|\boldsymbol{f}(\theta)-\boldsymbol{c}\|_{\mathbf{R}^{n}}^{4}}\right] \\
& =\frac{r^{2}}{\|\boldsymbol{f}(\theta)-\boldsymbol{c}\|_{\mathbf{R}^{n}}^{2}}\left(P_{c}^{\perp}(\theta)-P_{c}(\theta)\right) \dot{\boldsymbol{f}}(\theta),
\end{aligned}
$$

and therefore

$$
\begin{aligned}
\|\dot{\boldsymbol{p}}(\theta)\|_{\mathbf{R}^{n}}^{2} & =\frac{r^{4}}{\|\boldsymbol{f}(\theta)-\boldsymbol{c}\|_{\mathbf{R}^{n}}^{4}}\left(\left\|P_{c}^{\perp}(\theta) \dot{\boldsymbol{f}}(\theta)\right\|_{\mathbf{R}^{n}}^{2}+\left\|P_{c}(\theta) \dot{\boldsymbol{f}}(\theta)\right\|_{\mathbf{R}^{n}}^{2}\right) \\
& =\frac{r^{4}\|\dot{\boldsymbol{f}}(\theta)\|_{\mathbf{R}^{n}}^{2}}{\|\boldsymbol{f}(\theta)-\boldsymbol{c}\|_{\mathbf{R}^{n}}^{4}}
\end{aligned}
$$

Using straightforward considerations,

$$
\begin{aligned}
\frac{\left\|\dot{\boldsymbol{p}}\left(\theta_{1}\right)\right\|_{\mathbf{R}^{n}}\left\|\dot{\boldsymbol{p}}\left(\theta_{2}\right)\right\|_{\mathbf{R}^{n}}}{\left\|\boldsymbol{p}\left(\theta_{1}\right)-\boldsymbol{p}\left(\theta_{2}\right)\right\|_{\mathbf{R}^{n}}^{2}} & =\frac{r^{2}\left\|\dot{\boldsymbol{f}}\left(\theta_{1}\right)\right\|_{\mathbf{R}^{n}}}{\left\|\boldsymbol{f}\left(\theta_{1}\right)-\boldsymbol{c}\right\|_{\mathbf{R}^{n}}^{2}} \frac{r^{2}\left\|\dot{\boldsymbol{f}}\left(\theta_{2}\right)\right\|_{\mathbf{R}^{n}}}{\left\|\boldsymbol{f}\left(\theta_{2}\right)-\boldsymbol{c}\right\|_{\mathbf{R}^{n}}^{2}} \frac{\left\|\boldsymbol{f}\left(\theta_{1}\right)-\boldsymbol{c}\right\|_{\mathbf{R}^{n}}^{2}\left\|\boldsymbol{f}\left(\theta_{2}\right)-\boldsymbol{c}\right\|_{\mathbf{R}^{n}}^{2}}{r^{4}\left\|\boldsymbol{f}\left(\theta_{1}\right)-\boldsymbol{f}\left(\theta_{2}\right)\right\|_{\mathbf{R}^{n}}^{2}} \\
& =\frac{\left\|\dot{\boldsymbol{f}}\left(\theta_{1}\right)\right\|_{\mathbf{R}^{n}}\left\|\dot{\boldsymbol{f}}\left(\theta_{2}\right)\right\|_{\mathbf{R}^{n}}}{\left\|\boldsymbol{f}\left(\theta_{1}\right)-\boldsymbol{f}\left(\theta_{2}\right)\right\|_{\mathbf{R}^{n}}^{2}}
\end{aligned}
$$

and this demonstrates that $J_{1}=0 . \quad$ By a similar calculation

$$
\frac{\dot{\boldsymbol{p}}\left(\theta_{1}\right) \cdot \dot{\boldsymbol{p}}\left(\theta_{2}\right)}{\left\|\boldsymbol{p}\left(\theta_{1}\right)-\boldsymbol{p}\left(\theta_{2}\right)\right\|_{\mathbf{R}^{n}}^{2}}=\frac{\left\{\left(I-2 P_{c}\left(\theta_{1}\right)\right) \dot{\boldsymbol{f}}\left(\theta_{1}\right)\right\} \cdot\left\{\left(I-2 P_{c}\left(\theta_{2}\right)\right) \dot{\boldsymbol{f}}\left(\theta_{2}\right)\right\}}{\left\|\boldsymbol{f}\left(\theta_{1}\right)-\boldsymbol{f}\left(\theta_{2}\right)\right\|_{\mathbf{R}^{n}}^{2}}
$$

holds. Observing that

$$
\begin{aligned}
\left(I-2 P_{c}\left(\theta_{i}\right)\right) \dot{\boldsymbol{f}}\left(\theta_{i}\right) & =\dot{\boldsymbol{f}}\left(\theta_{i}\right)-2\left(\frac{\boldsymbol{f}\left(\theta_{i}\right)-\boldsymbol{c}}{\left\|\boldsymbol{f}\left(\theta_{i}\right)-\boldsymbol{c}\right\|_{\mathbf{R}^{n}}} \cdot \dot{\boldsymbol{f}}\left(\theta_{i}\right)\right) \frac{\boldsymbol{f}\left(\theta_{i}\right)-\boldsymbol{c}}{\left\|\boldsymbol{f}\left(\theta_{i}\right)-\boldsymbol{c}\right\|_{\mathbf{R}^{n}}} \\
& =\dot{\boldsymbol{f}}\left(\theta_{i}\right)-\left(\frac{\partial}{\partial \theta_{i}} \log \left\|\boldsymbol{f}\left(\theta_{i}\right)-\boldsymbol{c}\right\|_{\mathbf{R}^{n}}^{2}\right)\left(\boldsymbol{f}\left(\theta_{i}\right)-\boldsymbol{c}\right)
\end{aligned}
$$

we may write 


$$
\begin{gathered}
J_{2}=\frac{1}{\left\|\boldsymbol{f}\left(\theta_{1}\right)-\boldsymbol{f}\left(\theta_{2}\right)\right\|_{\mathbf{R}^{n}}^{2}} \\
\times\left[\dot{\boldsymbol{f}}\left(\theta_{1}\right) \cdot \dot{\boldsymbol{f}}\left(\theta_{2}\right)-\left\{\dot{\boldsymbol{f}}\left(\theta_{1}\right)-\left(\frac{\partial}{\partial \theta_{1}} \log \left\|\boldsymbol{f}\left(\theta_{1}\right)-\boldsymbol{c}\right\|_{\mathbf{R}^{n}}^{2}\right)\left(\boldsymbol{f}\left(\theta_{1}\right)-\boldsymbol{c}\right)\right\}\right. \\
\left.\cdot\left\{\dot{\boldsymbol{f}}\left(\theta_{2}\right)-\left(\frac{\partial}{\partial \theta_{1}} \log \left\|\boldsymbol{f}\left(\theta_{2}\right)-\boldsymbol{c}\right\|_{\mathbf{R}^{n}}^{2}\right)\left(\boldsymbol{f}\left(\theta_{2}\right)-\boldsymbol{c}\right)\right\}\right] \\
=\frac{1}{\left\|\boldsymbol{f}\left(\theta_{1}\right)-\boldsymbol{f}\left(\theta_{2}\right)\right\|_{\mathbf{R}^{n}}^{2}}\left[\left(\frac{\partial}{\partial \theta_{1}} \log \left\|\boldsymbol{f}\left(\theta_{1}\right)-\boldsymbol{c}\right\|_{\mathbf{R}^{n}}^{2}\right)\left\{\left(\boldsymbol{f}\left(\theta_{1}\right)-\boldsymbol{c}\right) \cdot \dot{\boldsymbol{f}}\left(\theta_{2}\right)\right\}\right. \\
\quad+\left(\frac{\partial}{\partial \theta_{2}} \log \left\|\boldsymbol{f}\left(\theta_{2}\right)-\boldsymbol{c}\right\|_{\mathbf{R}^{n}}^{2}\right)\left\{\left(\boldsymbol{f}\left(\theta_{2}\right)-\boldsymbol{c}\right) \cdot \dot{\boldsymbol{f}}\left(\theta_{1}\right)\right\} \\
\quad-\left(\frac{\partial}{\partial \theta_{1}} \log \left\|\boldsymbol{f}\left(\theta_{1}\right)-\boldsymbol{c}\right\|_{\mathbf{R}^{n}}^{2}\right)\left(\frac{\partial}{\partial \theta_{2}} \log \left\|\boldsymbol{f}\left(\theta_{2}\right)-\boldsymbol{c}\right\|_{\mathbf{R}^{n}}^{2}\right) \\
\left.\quad \times\left\{\left(\boldsymbol{f}\left(\theta_{1}\right)-\boldsymbol{c}\right) \cdot\left(\boldsymbol{f}\left(\theta_{2}\right)-\boldsymbol{c}\right)\right\}\right] .
\end{gathered}
$$

Using

$$
\begin{aligned}
\left(\boldsymbol{f}\left(\theta_{1}\right)-\boldsymbol{c}\right) \cdot \dot{\boldsymbol{f}}\left(\theta_{2}\right) & =\left(\boldsymbol{f}\left(\theta_{1}\right)-\boldsymbol{f}\left(\theta_{2}\right)+\boldsymbol{f}\left(\theta_{2}\right)-\boldsymbol{c}\right) \cdot \dot{\boldsymbol{f}}\left(\theta_{2}\right) \\
& =\frac{1}{2} \frac{\partial}{\partial \theta_{2}}\left(-\left\|\boldsymbol{f}\left(\theta_{1}\right)-\boldsymbol{f}\left(\theta_{2}\right)\right\|_{\mathbf{R}^{n}}^{2}+\left\|\boldsymbol{f}\left(\theta_{2}\right)-\boldsymbol{c}\right\|_{\mathbf{R}^{n}}^{2}\right)
\end{aligned}
$$

and

$$
\begin{aligned}
\left(\boldsymbol{f}\left(\theta_{2}\right)-\boldsymbol{c}\right) \cdot \dot{\boldsymbol{f}}\left(\theta_{1}\right) & =\left(\boldsymbol{f}\left(\theta_{2}\right)-\boldsymbol{f}\left(\theta_{1}\right)+\boldsymbol{f}\left(\theta_{1}\right)-\boldsymbol{c}\right) \cdot \dot{\boldsymbol{f}}\left(\theta_{1}\right) \\
& =\frac{1}{2} \frac{\partial}{\partial \theta_{1}}\left(-\left\|\boldsymbol{f}\left(\theta_{1}\right)-\boldsymbol{f}\left(\theta_{2}\right)\right\|_{\mathbf{R}^{n}}^{2}+\left\|\boldsymbol{f}\left(\theta_{1}\right)-\boldsymbol{c}\right\|_{\mathbf{R}^{n}}^{2}\right),
\end{aligned}
$$

we arrive at

$$
\begin{aligned}
\frac{\left(\boldsymbol{f}\left(\theta_{1}\right)-\boldsymbol{c}\right) \cdot \dot{\boldsymbol{f}}\left(\theta_{2}\right)}{\left\|\boldsymbol{f}\left(\theta_{1}\right)-\boldsymbol{f}\left(\theta_{2}\right)\right\|_{\mathbf{R}^{n}}^{2}}= & -\frac{1}{2} \frac{\partial}{\partial \theta_{2}} \log \left\|\boldsymbol{f}\left(\theta_{1}\right)-\boldsymbol{f}\left(\theta_{2}\right)\right\|_{\mathbf{R}^{n}}^{2} \\
& +\frac{1}{2} \frac{\left\|\boldsymbol{f}\left(\theta_{2}\right)-\boldsymbol{c}\right\|_{\mathbf{R}^{n}}^{2}}{\left\|\boldsymbol{f}\left(\theta_{1}\right)-\boldsymbol{f}\left(\theta_{2}\right)\right\|_{\mathbf{R}^{n}}^{2}} \frac{\partial}{\partial \theta_{2}} \log \left\|\boldsymbol{f}\left(\theta_{2}\right)-\boldsymbol{c}\right\|_{\mathbf{R}^{n}}^{2}, \\
\frac{\left(\boldsymbol{f}\left(\theta_{2}\right)-\boldsymbol{c}\right) \cdot \dot{\boldsymbol{f}}\left(\theta_{1}\right)}{\left\|\boldsymbol{f}\left(\theta_{1}\right)-\boldsymbol{f}\left(\theta_{2}\right)\right\|_{\mathbf{R}^{n}}^{2}}= & -\frac{1}{2} \frac{\partial}{\partial \theta_{1}} \log \left\|\boldsymbol{f}\left(\theta_{1}\right)-\boldsymbol{f}\left(\theta_{2}\right)\right\|_{\mathbf{R}^{n}}^{2} \\
& +\frac{1}{2} \frac{\left\|\boldsymbol{f}\left(\theta_{1}\right)-\boldsymbol{c}\right\|_{\mathbf{R}^{n}}^{2}}{\left\|\boldsymbol{f}\left(\theta_{1}\right)-\boldsymbol{f}\left(\theta_{2}\right)\right\|_{\mathbf{R}^{n}}^{2}} \frac{\partial}{\partial \theta_{1}} \log \left\|\boldsymbol{f}\left(\theta_{1}\right)-\boldsymbol{c}\right\|_{\mathbf{R}^{n}}^{2}
\end{aligned}
$$

upon which our previous expression for $J_{2}$ becomes 


$$
\begin{aligned}
J_{2}= & -\frac{1}{2}\left(\frac{\partial}{\partial \theta_{1}} \log \left\|\boldsymbol{f}\left(\theta_{1}\right)-\boldsymbol{c}\right\|_{\mathbf{R}^{n}}^{2}\right)\left(\frac{\partial}{\partial \theta_{2}} \log \left\|\boldsymbol{f}\left(\theta_{1}\right)-\boldsymbol{f}\left(\theta_{2}\right)\right\|_{\mathbf{R}^{n}}^{2}\right) \\
& -\frac{1}{2}\left(\frac{\partial}{\partial \theta_{1}} \log \left\|\boldsymbol{f}\left(\theta_{1}\right)-\boldsymbol{f}\left(\theta_{2}\right)\right\|_{\mathbf{R}^{n}}^{2}\right)\left(\frac{\partial}{\partial \theta_{2}} \log \left\|\boldsymbol{f}\left(\theta_{2}\right)-\boldsymbol{c}\right\|_{\mathbf{R}^{n}}^{2}\right) \\
& +\frac{1}{2}\left(\frac{\partial}{\partial \theta_{1}} \log \left\|\boldsymbol{f}\left(\theta_{1}\right)-\boldsymbol{c}\right\|_{\mathbf{R}^{n}}^{2}\right)\left(\frac{\partial}{\partial \theta_{2}} \log \left\|\boldsymbol{f}\left(\theta_{2}\right)-\boldsymbol{c}\right\|_{\mathbf{R}^{n}}^{2}\right) \\
& \times \frac{\left\|\boldsymbol{f}\left(\theta_{2}\right)-\boldsymbol{c}\right\|_{\mathbf{R}^{n}}^{2}+\left\|\boldsymbol{f}\left(\theta_{1}\right)-\boldsymbol{c}\right\|_{\mathbf{R}^{n}}^{2}-2\left(\boldsymbol{f}\left(\theta_{1}\right)-\boldsymbol{c}\right) \cdot\left(\boldsymbol{f}\left(\theta_{2}\right)-\boldsymbol{c}\right)}{\left\|\boldsymbol{f}\left(\theta_{1}\right)-\boldsymbol{f}\left(\theta_{2}\right)\right\|_{\mathbf{R}^{n}}^{2}} \\
= & -\frac{1}{2}\left(\frac{\partial}{\partial \theta_{1}} \log \left\|\boldsymbol{f}\left(\theta_{1}\right)-\boldsymbol{c}\right\|_{\mathbf{R}^{n}}^{2}\right)\left(\frac{\partial}{\partial \theta_{2}} \log \left\|\boldsymbol{f}\left(\theta_{1}\right)-\boldsymbol{f}\left(\theta_{2}\right)\right\|_{\mathbf{R}^{n}}^{2}\right) \\
& -\frac{1}{2}\left(\frac{\partial}{\partial \theta_{1}} \log \left\|\boldsymbol{f}\left(\theta_{1}\right)-\boldsymbol{f}\left(\theta_{2}\right)\right\|_{\mathbf{R}^{n}}^{2}\right)\left(\frac{\partial}{\partial \theta_{2}} \log \left\|\boldsymbol{f}\left(\theta_{2}\right)-\boldsymbol{c}\right\|_{\mathbf{R}^{n}}^{2}\right) \\
& +\frac{1}{2}\left(\frac{\partial}{\partial \theta_{1}} \log \left\|\boldsymbol{f}\left(\theta_{1}\right)-\boldsymbol{c}\right\|_{\mathbf{R}^{n}}^{2}\right)\left(\frac{\partial}{\partial \theta_{2}} \log \left\|\boldsymbol{f}\left(\theta_{2}\right)-\boldsymbol{c}\right\|_{\mathbf{R}^{n}}^{2}\right) .
\end{aligned}
$$

Finally,

$$
\frac{\partial}{\partial \theta_{i}} \log \left\|\boldsymbol{p}\left(\theta_{1}\right)-\boldsymbol{p}\left(\theta_{2}\right)\right\|_{\mathbf{R}^{n}}^{2}=\frac{\partial}{\partial \theta_{i}}\left(\log \left\|\boldsymbol{f}\left(\theta_{1}\right)-\boldsymbol{f}\left(\theta_{2}\right)\right\|_{\mathbf{R}^{n}}^{2}-\log \left\|\boldsymbol{f}\left(\theta_{i}\right)-\boldsymbol{c}\right\|_{\mathbf{R}^{n}}^{2}\right),
$$

and therefore

$$
\begin{aligned}
J_{3}= & \frac{1}{2}\left(\frac{\partial}{\partial \theta_{1}} \log \left\|\boldsymbol{f}\left(\theta_{1}\right)-\boldsymbol{c}\right\|_{\mathbf{R}^{n}}^{2}\right)\left(\frac{\partial}{\partial \theta_{2}} \log \left\|\boldsymbol{f}\left(\theta_{1}\right)-\boldsymbol{f}\left(\theta_{2}\right)\right\|_{\mathbf{R}^{n}}^{2}\right) \\
& +\frac{1}{2}\left(\frac{\partial}{\partial \theta_{1}} \log \left\|\boldsymbol{f}\left(\theta_{1}\right)-\boldsymbol{f}\left(\theta_{2}\right)\right\|_{\mathbf{R}^{n}}^{2}\right)\left(\frac{\partial}{\partial \theta_{2}} \log \left\|\boldsymbol{f}\left(\theta_{2}\right)-\boldsymbol{c}\right\|_{\mathbf{R}^{n}}^{2}\right) \\
& -\frac{1}{2}\left(\frac{\partial}{\partial \theta_{1}} \log \left\|\boldsymbol{f}\left(\theta_{1}\right)-\boldsymbol{c}\right\|_{\mathbf{R}^{n}}^{2}\right)\left(\frac{\partial}{\partial \theta_{2}} \log \left\|\boldsymbol{f}\left(\theta_{2}\right)-\boldsymbol{c}\right\|_{\mathbf{R}^{n}}^{2}\right) \\
= & -J_{2} .
\end{aligned}
$$

COROllary 3.1. Let $\boldsymbol{f} \in H^{1,1}(\mathbf{R} / 2 \pi \mathbf{Z})$. Then it holds that

$$
\mathcal{E}_{1}(\boldsymbol{f})+\mathcal{E}_{2}(\boldsymbol{f})=\mathcal{E}_{1}(\boldsymbol{p})+\mathcal{E}_{2}(\boldsymbol{p}) .
$$

Remark 3.1. The corollary does not exclude that both sides are infinite. of

Proof. First let $\boldsymbol{f} \in H^{3 / 2}(\mathbf{R} / 2 \pi \mathbf{Z})$. If the 2-dimensional Lebesgue measure

$$
\left\{\left(\theta_{1}, \theta_{2}\right) \in(\mathbf{R} / 2 \pi \mathbf{Z})^{2} \mid \boldsymbol{f}\left(\theta_{1}\right)=\boldsymbol{f}\left(\theta_{2}\right)\right\}
$$


is positive, then so is that of

$$
\left\{\left(\theta_{1}, \theta_{2}\right) \in(\mathbf{R} / 2 \pi \mathbf{Z})^{2} \mid \boldsymbol{p}\left(\theta_{1}\right)=\boldsymbol{p}\left(\theta_{2}\right)\right\} .
$$

Therefore $\mathcal{E}(\boldsymbol{f})=\mathcal{E}(\boldsymbol{p})=\infty$ and we have

$$
\mathcal{E}_{1}(\boldsymbol{f})+\mathcal{E}_{2}(\boldsymbol{f})=\mathcal{E}_{1}(\boldsymbol{p})+\mathcal{E}_{2}(\boldsymbol{p})=\infty .
$$

Thus, we assume that the 2-dimensional Lebesgue measure of

$$
\left\{\left(\theta_{1}, \theta_{2}\right) \in(\mathbf{R} / 2 \pi \mathbf{Z})^{2} \mid \boldsymbol{f}\left(\theta_{1}\right)=\boldsymbol{f}\left(\theta_{2}\right)\right\}
$$

is 0 , and we claim that the measure of

$$
\left\{\left(\theta_{1}, \theta_{2}\right) \in(\mathbf{R} / 2 \pi \mathbf{Z})^{2} \mid \boldsymbol{f}\left(\theta_{1}\right)=\boldsymbol{f}\left(\theta_{2}\right) \text { or } \boldsymbol{f}\left(\theta_{1}\right)=\boldsymbol{c} \text { or } \boldsymbol{f}\left(\theta_{2}\right)=\boldsymbol{c}\right\}
$$

is also 0 . In order to see this, considering $f$ as a function of $s$ on $\mathbf{R} / \mathcal{L Z}$, we need to prove that

$$
S=\{s \in \mathbf{R} / \mathcal{L} \mathbf{Z} \mid \boldsymbol{f}(s)=\boldsymbol{c}\}
$$

is a finite set. Arguing by contradiction, we suppose that $S$ is not a finite set and using the compactness of $\mathbf{R} / \mathcal{L} \mathbf{Z}$, there exists a sequence such that $\boldsymbol{f}\left(s_{j}\right)=\boldsymbol{c}$ and $\lim _{j \rightarrow \infty} s_{j}=s_{*}$. From

$$
\begin{aligned}
0 & =\left\|\boldsymbol{f}\left(s_{j+1}\right)-\boldsymbol{f}\left(s_{j}\right)\right\|_{\mathbf{R}^{n}}^{2}=\int_{s_{j}}^{s_{j+1}} \int_{s_{j}}^{s_{j+1}} \boldsymbol{\tau}(s) \cdot \boldsymbol{\tau}\left(s^{\prime}\right) d s d s^{\prime} \\
& =\left(s_{j+1}-s_{j}\right)^{2}-\frac{1}{2} \int_{s_{j}}^{s_{j+1}} \int_{s_{j}}^{s_{j+1}}\left\|\boldsymbol{\tau}(s)-\boldsymbol{\tau}\left(s^{\prime}\right)\right\|_{\mathbf{R}^{n}}^{2} d s d s^{\prime},
\end{aligned}
$$

it holds that

$$
\int_{s_{j}}^{s_{j+1}} \int_{s_{j}}^{s_{j+1}} \frac{\left\|\boldsymbol{\tau}(s)-\boldsymbol{\tau}\left(s^{\prime}\right)\right\|_{\mathbf{R}^{n}}^{2}}{\left(s-s^{\prime}\right)^{2}} d s d s^{\prime} \geq \int_{s_{j}}^{s_{j+1}} \int_{s_{j}}^{s_{j+1}} \frac{\left\|\boldsymbol{\tau}(s)-\boldsymbol{\tau}\left(s^{\prime}\right)\right\|_{\mathbf{R}^{n}}^{2}}{\left(s_{j+1}-s_{j}\right)^{2}} d s d s^{\prime}=2 .
$$

However, using that $\tau \in H^{1 / 2}(\mathbf{R} / \mathcal{L} \mathbf{Z})$ and the absolute continuity of integral, this leads to

$$
2 \leq \lim _{j \rightarrow \infty} \int_{s_{j}}^{s_{j+1}} \int_{s_{j}}^{s_{j+1}} \frac{\left\|\boldsymbol{\tau}(s)-\boldsymbol{\tau}\left(s^{\prime}\right)\right\|_{\mathbf{R}^{n}}^{2}}{\left(s-s^{\prime}\right)^{2}} d s d s^{\prime}=0,
$$

which is obviously a contradiction. By these arguments we find that (6) holds for $\mathscr{L}^{2}$-a.e. $\left(\theta_{1}, \theta_{2}\right)$ and the desired conclusion follows by integrating this.

Finally, we consider the case of $\boldsymbol{f} \notin H^{3 / 2}(\mathbf{R} / 2 \pi \mathbf{Z})$ which implies $\mathcal{E}(\boldsymbol{f})=\infty$, and we will show $\mathcal{E}(\boldsymbol{p})=\infty$. Again, arguing by contradiction, we suppose that 
$\mathcal{E}(\boldsymbol{p})<\infty$ from which we know that $\boldsymbol{p}$ does not have self-intersections. Furthermore $\boldsymbol{p} \in H_{\text {loc }}^{3 / 2}$ and we remark that $\boldsymbol{p} \in H^{3 / 2}$ if $\boldsymbol{p}$ does not pass through the point at infinity. If we turn $\boldsymbol{p}$ back by the inversion with respect to the sphere with center $c$ and radius $r$, then it returns to $f$. Since $f$ does not pass through the point at infinity, $\boldsymbol{p}$ does not pass through $\boldsymbol{c}$. Thereby the 2-dimensional Lebesgue measure of

$$
\left\{\left(\theta_{1}, \theta_{2}\right) \mid \boldsymbol{p}\left(\theta_{1}\right)=\boldsymbol{p}\left(\theta_{2}\right), \text { or } \boldsymbol{p}\left(\theta_{1}\right)=\boldsymbol{c} \text { or } \boldsymbol{p}\left(\theta_{2}\right)=\boldsymbol{c}\right\}
$$

is 0 . This implies that $(6)$ holds for $\mathscr{L}^{2}$-a.e. $\left(\theta_{1}, \theta_{2}\right)$ and integrating this, we get

$$
\mathcal{E}_{1}(\boldsymbol{f})+\mathcal{E}_{2}(\boldsymbol{f})=\mathcal{E}_{1}(\boldsymbol{p})+\mathcal{E}_{2}(\boldsymbol{p})=\mathcal{E}(\boldsymbol{p})-4<\infty .
$$

However, from this we obtain $\infty=\mathcal{E}(f)=\mathcal{E}_{1}(f)+\mathcal{E}_{2}(f)+4<\infty$ which is obviously a contradiction. As a conclusion, $\mathcal{E}(p)=\infty$ holds and therefore $\mathcal{E}_{1}(f)+\mathcal{E}_{2}(f)=\mathcal{E}_{1}(p)+\mathcal{E}_{2}(p)=\infty$ as desired.

\subsection{The invariance of each energy}

We discuss here the invariance of each energy $\mathcal{E}_{i}$ under the inversion $\boldsymbol{f} \mapsto \boldsymbol{p}$.

THEOREM 3.2. Assume that the center $c$ of the inversion is not in the image of $\boldsymbol{f}$. We also assume the finiteness of energy $\mathcal{E}(\boldsymbol{f})<\infty$. Then

$$
\mathcal{E}_{1}(\boldsymbol{f})=\mathcal{E}_{1}(\boldsymbol{p}), \quad \mathcal{E}_{2}(\boldsymbol{f})=\mathcal{E}_{2}(\boldsymbol{p})
$$

holds.

Proof. In view of Corollary 3.1, it is enough to prove $\mathcal{E}_{1}(\boldsymbol{f})=\mathcal{E}_{1}(\boldsymbol{p})$. Let $J_{1}$ and $J_{2}$ be as defined in the proof of Theorem 3.1. It follows that

$$
\mathscr{M}_{1}(\boldsymbol{f})\left\|\dot{\boldsymbol{f}}\left(\theta_{1}\right)\right\|_{\mathbf{R}^{n}}\left\|\dot{\boldsymbol{f}}\left(\theta_{2}\right)\right\|_{\mathbf{R}^{n}}-\mathscr{M}_{1}(\boldsymbol{p})\left\|\dot{\boldsymbol{p}}\left(\theta_{1}\right)\right\|_{\mathbf{R}^{n}}\left\|\dot{\boldsymbol{p}}\left(\theta_{2}\right)\right\|_{\mathbf{R}^{n}}=J_{1}-J_{2}=-J_{2} .
$$

We need to prove that the integration of this goes to 0 . From now on, we use the arc-length variable $s_{j}$.

Since we are assuming $\mathcal{E}(\boldsymbol{f})<\infty$, we know that $\mathscr{M}_{1}(\boldsymbol{f}) \in L^{1}\left((\mathbf{R} / \mathcal{L} \mathbf{Z})^{2}\right)$ holds. Since $\mathscr{M}_{1}(\boldsymbol{p}) \geq 0$, we may write

$$
\mathcal{E}_{1}(\boldsymbol{f})-\mathcal{E}_{1}(\boldsymbol{p})=-\lim _{\varepsilon \rightarrow+0} \iint_{\left|s_{1}-s_{2}\right| \geq \varepsilon} \frac{J_{2}}{\left\|\dot{\boldsymbol{f}}\left(\theta_{1}\right)\right\|_{\mathbf{R}^{n}}\left\|\dot{\boldsymbol{f}}\left(\theta_{2}\right)\right\|_{\mathbf{R}^{n}}} d s_{1} d s_{2},
$$

where $\left|s_{1}-s_{2}\right| \geq \varepsilon$ is in the sense of $\bmod \mathcal{L}$. Remarking that

$$
\frac{1}{\left\|\dot{\boldsymbol{f}}\left(\theta_{j}\right)\right\|_{\mathbf{R}^{n}}} \frac{\partial}{\partial \theta_{j}}(\cdots)=\frac{\partial}{\partial s_{j}}(\cdots)
$$

we get 


$$
\begin{aligned}
-\lim _{\varepsilon \rightarrow+0} & \iint_{\left|s_{1}-s_{2}\right| \geq \varepsilon} \frac{J_{2}}{\left\|\dot{\boldsymbol{f}}\left(\theta_{1}\right)\right\|_{\mathbf{R}^{n}}\left\|\dot{\boldsymbol{f}}\left(\theta_{2}\right)\right\|_{\mathbf{R}^{n}}} d s_{1} d s_{2} \\
= & \lim _{\varepsilon \rightarrow+0} \frac{1}{2} \iint_{\left|s_{1}-s_{2}\right| \geq \varepsilon}\left\{\left(\frac{\partial}{\partial s_{1}} \log \left\|\boldsymbol{f}\left(s_{1}\right)-\boldsymbol{c}\right\|_{\mathbf{R}^{n}}^{2}\right)\left(\frac{\partial}{\partial s_{2}} \log \left\|\boldsymbol{f}\left(s_{1}\right)-\boldsymbol{f}\left(s_{2}\right)\right\|_{\mathbf{R}^{n}}^{2}\right)\right. \\
& +\left(\frac{\partial}{\partial s_{1}} \log \left\|\boldsymbol{f}\left(s_{1}\right)-\boldsymbol{f}\left(s_{2}\right)\right\|_{\mathbf{R}^{n}}^{2}\right)\left(\frac{\partial}{\partial s_{2}} \log \left\|\boldsymbol{f}\left(s_{2}\right)-\boldsymbol{c}\right\|_{\mathbf{R}^{n}}^{2}\right) \\
& \left.-\left(\frac{\partial}{\partial s_{1}} \log \left\|\boldsymbol{f}\left(s_{1}\right)-\boldsymbol{c}\right\|_{\mathbf{R}^{n}}^{2}\right)\left(\frac{\partial}{\partial \theta_{2}} \log \left\|\boldsymbol{f}\left(s_{2}\right)-\boldsymbol{c}\right\|_{\mathbf{R}^{n}}^{2}\right)\right\} d s_{1} d s_{2} \\
= & \lim _{\varepsilon \rightarrow+0} \frac{1}{2} \int_{0}^{\mathcal{L}} \frac{\partial}{\partial s_{1}} \log \left\|\boldsymbol{f}\left(s_{1}\right)-\boldsymbol{c}\right\|_{\mathbf{R}^{n}}^{2} \int_{s_{1}+\varepsilon}^{s_{1}+\mathcal{L}-\varepsilon} \frac{\partial}{\partial s_{2}} \log \left\|\boldsymbol{f}\left(s_{1}\right)-\boldsymbol{f}\left(s_{2}\right)\right\|_{\mathbf{R}^{n}}^{2} d s_{2} d s_{1} \\
& +\lim _{\varepsilon \rightarrow+0} \frac{1}{2} \int_{0}^{\mathcal{L}} \frac{\partial}{\partial s_{2}} \log \left\|\boldsymbol{f}\left(s_{2}\right)-\boldsymbol{c}\right\|_{\mathbf{R}^{n}}^{2} \int_{s_{2}+\varepsilon}^{s_{2}+\mathcal{L}-\varepsilon} \frac{\partial}{\partial s_{1}} \log \left\|\boldsymbol{f}\left(s_{1}\right)-\boldsymbol{f}\left(s_{2}\right)\right\|_{\mathbf{R}^{n}}^{2} d s_{1} d s_{2} \\
& -\frac{1}{2} \int_{0}^{\mathcal{L}} \frac{\partial}{\partial s_{1}} \log \left\|\boldsymbol{f}\left(s_{1}\right)-\boldsymbol{c}\right\|_{\mathbf{R}^{n}}^{2} d s_{1} \int_{0}^{\mathcal{L}} \frac{\partial}{\partial \theta_{2}} \log \left\|\boldsymbol{f}\left(s_{2}\right)-\boldsymbol{c}\right\|_{\mathbf{R}^{n}}^{2} d s_{2} .
\end{aligned}
$$

It holds that

$$
\int_{s_{1}+\varepsilon}^{s_{1}+\mathcal{L}-\varepsilon} \frac{\partial}{\partial s_{2}} \log \left\|\boldsymbol{f}\left(s_{1}\right)-\boldsymbol{f}\left(s_{2}\right)\right\|_{\mathbf{R}^{n}}^{2} d s_{2}=\log \frac{\left\|\boldsymbol{f}\left(s_{1}\right)-\boldsymbol{f}\left(s_{1}-\varepsilon\right)\right\|_{\mathbf{R}^{n}}^{2}}{\left\|\boldsymbol{f}\left(s_{1}\right)-\boldsymbol{f}\left(s_{1}+\varepsilon\right)\right\|_{\mathbf{R}^{n}}^{2}} .
$$

Moreover, $\mathcal{E}(\boldsymbol{f})<\infty$ implies that $\boldsymbol{f} \in H^{3 / 2}(\mathbf{R} / \mathcal{L Z})$ and thus

$$
\left\|\boldsymbol{f}\left(s_{1}\right)-\boldsymbol{f}\left(s_{1} \pm \varepsilon\right)\right\|_{\mathbf{R}^{n}}^{2}=\varepsilon^{2}+o\left(\varepsilon^{2}\right)
$$

uniformly with regard to $s_{1}$ as $\varepsilon \rightarrow+0$. Therefore

$$
\int_{s_{1}+\varepsilon}^{s_{1}+\mathcal{L}-\varepsilon} \frac{\partial}{\partial s_{2}} \log \left\|\boldsymbol{f}\left(s_{1}\right)-\boldsymbol{f}\left(s_{2}\right)\right\|_{\mathbf{R}^{n}}^{2} d s_{2}=\log (1+o(1))=o(1)
$$

holds uniformly with regard to $s_{1}$. From this and the fact that $f$ does not pass through $c$,

$$
\begin{aligned}
& \left|\int_{0}^{\mathcal{L}} \frac{\partial}{\partial s_{1}} \log \left\|\boldsymbol{f}\left(s_{1}\right)-\boldsymbol{c}\right\|_{\mathbf{R}^{n}}^{2} \int_{s_{1}+\varepsilon}^{s_{1}+\mathcal{L}-\varepsilon} \frac{\partial}{\partial s_{2}} \log \left\|\boldsymbol{f}\left(s_{1}\right)-\boldsymbol{f}\left(s_{2}\right)\right\|_{\mathbf{R}^{n}}^{2} d s_{2} d s_{1}\right| \\
& \quad=o(1) \int_{0}^{\mathcal{L}}\left|\frac{\partial}{\partial s_{1}} \log \left\|\boldsymbol{f}\left(s_{1}\right)-\boldsymbol{c}\right\|_{\mathbf{R}^{n}}^{2}\right| d s_{1} \\
& \quad=o(1)
\end{aligned}
$$

holds. Then we arrive at

$$
\lim _{\varepsilon \rightarrow+0} \int_{0}^{\mathcal{L}} \frac{\partial}{\partial s_{1}} \log \left\|\boldsymbol{f}\left(s_{1}\right)-\boldsymbol{c}\right\|_{\mathbf{R}^{n}}^{2} \int_{s_{1}+\varepsilon}^{s_{1}+\mathcal{L}-\varepsilon} \frac{\partial}{\partial s_{2}} \log \left\|\boldsymbol{f}\left(s_{1}\right)-\boldsymbol{f}\left(s_{2}\right)\right\|_{\mathbf{R}^{n}}^{2} d s_{2} d s_{1}=0
$$


and similarly we find that

$$
\lim _{\varepsilon \rightarrow+0} \int_{0}^{\mathcal{L}} \frac{\partial}{\partial s_{2}} \log \left\|\boldsymbol{f}\left(s_{2}\right)-\boldsymbol{c}\right\|_{\mathbf{R}^{n}}^{2} \int_{s_{2}+\varepsilon}^{s_{2}+\mathcal{L}-\varepsilon} \frac{\partial}{\partial s_{1}} \log \left\|\boldsymbol{f}\left(s_{1}\right)-\boldsymbol{f}\left(s_{2}\right)\right\|_{\mathbf{R}^{n}}^{2} d s_{1} d s_{2}=0 .
$$

Finally, since $f$ does not pass through $\boldsymbol{c}$, we have

$$
\int_{0}^{\mathcal{L}} \frac{\partial}{\partial s_{1}} \log \left\|\boldsymbol{f}\left(s_{1}\right)-\boldsymbol{c}\right\|_{\mathbf{R}^{n}}^{2} d s_{1} \int_{0}^{\mathcal{L}} \frac{\partial}{\partial \theta_{2}} \log \left\|\boldsymbol{f}\left(s_{2}\right)-\boldsymbol{c}\right\|_{\mathbf{R}^{n}}^{2} d s_{2}=0
$$

from which the desired conclusion $\mathcal{E}_{1}(\boldsymbol{f})=\mathcal{E}(\boldsymbol{p})$ follows.

Remark 3.2. If $\boldsymbol{f}$ passes through $\boldsymbol{c}$, then $\mathcal{E}_{1}(\boldsymbol{f})=\mathcal{E}_{1}(\boldsymbol{p})$ does not hold. For example, when $\boldsymbol{f}$ is a circle passing through $\boldsymbol{c}$, then $\boldsymbol{p}$ is a straight line. If $\rho$ denotes the radius of $f$, then

$$
\rho\left\|\boldsymbol{\tau}\left(s_{1}\right)-\boldsymbol{\tau}\left(s_{2}\right)\right\|_{\mathbf{R}^{n}}=\left\|\boldsymbol{f}\left(s_{1}\right)-\boldsymbol{f}\left(s_{2}\right)\right\|_{\mathbf{R}^{n}}
$$

and

$$
\mathcal{E}_{1}(\boldsymbol{f})=\iint_{(\mathbf{R} / \mathcal{L} \mathbf{Z})^{2}} \frac{\left\|\boldsymbol{f}\left(s_{1}\right)-\boldsymbol{f}\left(s_{2}\right)\right\|_{\mathbf{R}^{n}}^{2}}{2 \rho^{2}\left\|\boldsymbol{f}\left(s_{1}\right)-\boldsymbol{f}\left(s_{2}\right)\right\|_{\mathbf{R}^{n}}^{2}} d s_{1} d s_{2}=\frac{(2 \pi \rho)^{2}}{2 \rho^{2}}=2 \pi^{2}
$$

hold. On the other hand, the unit tangent vector of $\boldsymbol{p}$ is a constant vector. This means $\mathscr{M}_{1}(\boldsymbol{p}) \equiv 0$ and consequently $\mathcal{E}_{1}(\boldsymbol{p})=0$.

Remark 3.3. In general, if $\boldsymbol{c}$ is in the image of $\boldsymbol{f}$, then

$$
\mathcal{E}_{1}(\boldsymbol{p})=\mathcal{E}_{1}(\boldsymbol{f})-2 \pi^{2}, \quad \mathcal{E}_{2}(\boldsymbol{p})=\mathcal{E}_{2}(\boldsymbol{f})+2 \pi^{2}
$$

seem to hold. Indeed we can show this fact if $\boldsymbol{f} \in C^{1,1}(\mathbf{R} / \mathcal{L} \mathbf{Z})$ and $\| \boldsymbol{f}\left(s_{1}\right)-$ $\boldsymbol{f}\left(s_{2}\right) \|_{\mathbf{R}^{n}} \geq \lambda^{-1} \mathscr{D}\left(\boldsymbol{f}\left(s_{1}\right), \boldsymbol{f}\left(s_{2}\right)\right)$ for some positive constant $\lambda$ independent of $s_{1}$ and $s_{2}$. The proof, however, needs quite long calculations, and therefore we present it elsewhere.

\section{REFERENCES}

[ 1 ] S. BLatT, Boundedness and regularizing effects of O'Hara's knot energies, J. Knot Theory Ramifications 21-1 (2012), 1250010.

[2] S. Blatt and P. Reiter, Stationary points of O'Hara's knot energy, Manuscripta Math. 140 (2013), 29-50.

[ 3 ] M. H. Freedman, Z.-X. He and Z. Wang, Möbius energy of knots and unknots, Ann. of Math. (2) 139 (1994), 1-50.

[4] A. Ishizeki, Some properties of the Möbius energy and its variational formulae, Master thesis, Saitama University, 2013.

[5] R. Kusner AND J. M. Sullivan, Möbius-invariant knot energies, Ideal knots, Ser. knots everything 19, World Sci. Publ., River Edge, NJ, 1998, 315-352.

[6] S. Mizohata, The theory of partial differential equations, Cambridge University Press, 1973.

[7] J. O'Hara, Energy of a knot, Topology 30 (1991), 241-247. 
[8] J. O'Hara and G. Solanes, Möbius invariant energies and average linking with circles, to appear in Tôhoku Math. J. (2).

Aya Ishizeki

Department of Mathematics

Graduate School of Science and Engineering

SAITAMa University

SAITAMa $338-8570$

JAPAN

E-mail: s13dm003@mail.saitama-u.ac.jp

Takeyuki Nagasawa

Department of Mathematics

Graduate School of Science and Engineering

SaItama University

SaITAMa $338-8570$

JAPAN

E-mail: tnagasaw@rimath.saitama-u.ac.jp 